Keywords: Tank Farm, Sludge, Waste, Chemistry, Performance Assessment, Technetium

Retention: Permanent

\title{
Chemical Differences Between Sludge Solids at the $F$ and $H$ Area Tank Farms
}

S. H. Reboul

August 2012

Savannah River National Laboratory Savannah River Nuclear Solutions, LLC Aiken, SC 29808

Prepared for the U.S. Department of Energy under contract number DE-AC09-08SR22470.

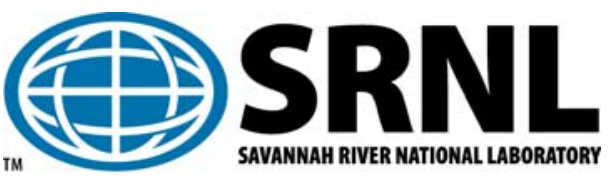


SRNL-STI-2012-00479

Revision 0

\section{DISCLAIMER}

This work was prepared under an agreement with and funded by the U.S. Government. Neither the U.S. Government or its employees, nor any of its contractors, subcontractors or their employees, makes any express or implied:

1. warranty or assumes any legal liability for the accuracy, completeness, or for the use or results of such use of any information, product, or process disclosed; or

2. representation that such use or results of such use would not infringe privately owned rights; or

3. endorsement or recommendation of any specifically identified commercial product, process, or service.

Any views and opinions of authors expressed in this work do not necessarily state or reflect those of the United States Government, or its contractors, or subcontractors.

\section{Printed in the United States of America \\ Prepared for \\ U.S. Department of Energy}




\section{REVIEWS AND APPROVALS}

AUTHORS:

S. H. Reboul, Process Technology Programs

Date

TECHNICAL REVIEW:

D. T. Hobbs, Separation \& Actinide Science Programs

Date

APPROVAL:

F. M. Pennebaker, Manager

Date

Advanced Characterization and Processing

H. H. Burns, Environmental \& Chemical Process Technology Programs Date

S.L. Marra, Manager

Date

Environmental \& Chemical Process Technology Research Programs

K. H. Rosenberger, Manager

Date

SRR Closure \& Waste Disposal Authority 


\section{EXECUTIVE SUMMARY}

The primary source of waste solids received into the F Area Tank Farm (FTF) was from PUREX processing performed to recover uranium and plutonium from irradiated depleted uranium targets. In contrast, two primary sources of waste solids were received into the H Area Tank Farm (HTF): a) waste from PUREX processing; and b) waste from $\mathrm{H}$-modified (HM) processing performed to recover uranium and neptunium from burned enriched uranium fuel. Due to the differences between the irradiated depleted uranium targets and the burned enriched uranium fuel, the average compositions of the $\mathrm{F}$ and $\mathrm{H}$ Area wastes are markedly different from one another.

Both $\mathrm{F}$ and $\mathrm{H}$ Area wastes contain significant amounts of iron and aluminum compounds. However, because the iron content of PUREX waste is higher than that of HM waste, and the aluminum content of PUREX waste is lower than that of HM waste, the iron to aluminum ratios of typical FTF waste solids are appreciably higher than those of typical HTF waste solids. Other constituents present at significantly higher concentrations in the typical FTF waste solids include uranium, nickel, ruthenium, zinc, silver, cobalt and copper. In contrast, constituents present at significantly higher concentrations in the typical HTF waste solids include mercury, thorium, oxalate, and radionuclides U-233, U-234, U-235, U-236, Pu-238, Pu-242, Cm-244, and Cm-245. Because of the higher concentrations of $\mathrm{Pu}-238$ in $\mathrm{HTF}$, the long-term concentrations of Th-230 and Ra-226 (from $\mathrm{Pu}-238$ decay) will also be higher in HTF.

The uranium and plutonium distributions of the average FTF waste were found to be consistent with depleted uranium and weapons grade plutonium, respectively (U-235 comprised $0.3 \mathrm{wt} \%$ of the FTF uranium, and $\mathrm{Pu}-240$ comprised $6 \mathrm{wt} \%$ of the FTF plutonium). In contrast, at HTF, U-235 comprised $5 \mathrm{wt} \%$ of the uranium, and $\mathrm{Pu}-240$ comprised $17 \mathrm{wt} \%$ of the plutonium, consistent with enriched uranium and high burn-up plutonium.

X-ray diffraction analyses of various FTF and HTF samples indicated that the primary crystalline compounds of iron in sludge solids are $\mathrm{Fe}_{2} \mathrm{O}_{3}, \mathrm{Fe}_{3} \mathrm{O}_{4}$, and $\mathrm{FeO}(\mathrm{OH})$, and the primary crystalline compounds of aluminum are $\mathrm{Al}(\mathrm{OH})_{3}$ and $\mathrm{AlO}(\mathrm{OH})$. Also identified were carbonate compounds of calcium, magnesium, and sodium; a nitrated sodium aluminosilicate; and various uranium compounds. Consistent with expectations, oxalate compounds were identified in solids associated with oxalic acid cleaning operations.

The most likely oxidation states and chemical forms of technetium are assessed in the context of solubility, since technetium-99 is a key risk driver from an environmental fate and transport perspective. The primary oxidation state of technetium in SRS sludge solids is expected to be Tc(IV). In salt waste, the primary oxidation state is expected to be Tc(VII). The primary form of technetium in sludge is expected to be a hydrated technetium dioxide, $\mathrm{TcO}_{2} \cdot \mathrm{xH}_{2} \mathrm{O}$, which is relatively insoluble and likely co-precipitated with iron. In salt waste solutions, the primary form of technetium is expected to be the very soluble pertechnetate anion, $\mathrm{TcO}_{4}^{-}$. 
The relative differences between the $\mathrm{F}$ and $\mathrm{H}$ Tank Farm waste provide a basis for anticipating differences that will occur as constituents of FTF and HTF waste residue enter the environment over the long-term future. If a constituent is significantly more dominant in one of the Tank Farms, its long-term environmental contribution will likely be commensurately higher, assuming the environmental transport conditions of the two Tank Farms share some commonality. It is in this vein that the information cited in this document is provided - for use during the generation, assessment, and validation of Performance Assessment modeling results. 


\section{TABLE OF CONTENTS}

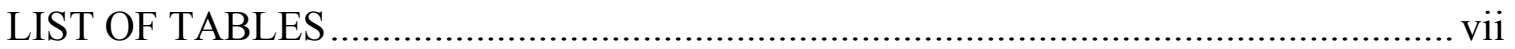

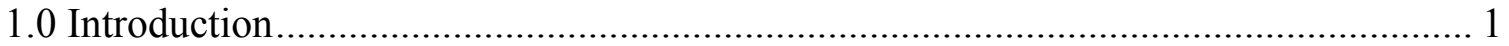

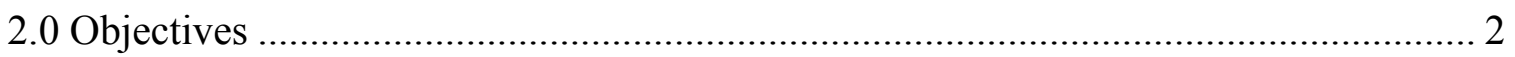

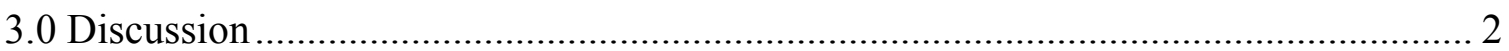

3.1 F and $\mathrm{H}$ Area Nuclear Materials Processing and Waste Characteristics................... 2

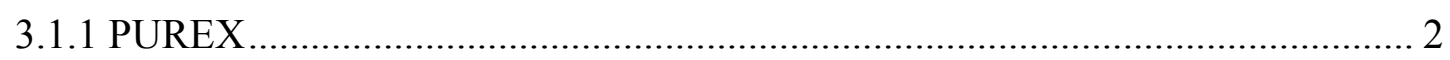

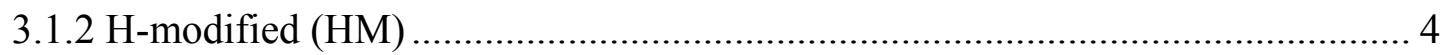

3.2 F and H Area Tank Farm Waste Receipts ................................................................. 5

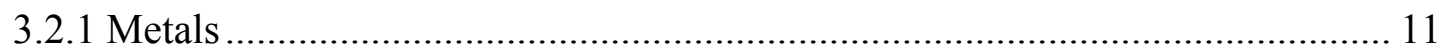

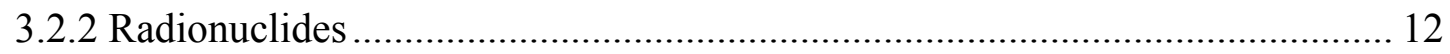

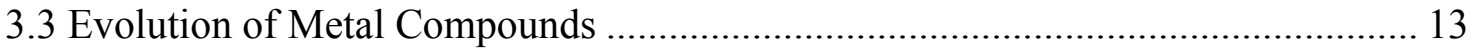

3.4 Mineral Phases in F and H Area Tank Farm Waste Solids .................................... 17

3.5 Solid/Liquid Phase Technetium Partitioning............................................................ 19

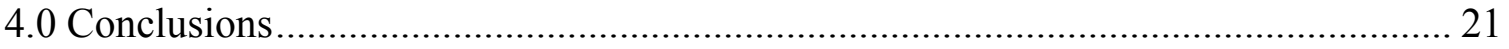

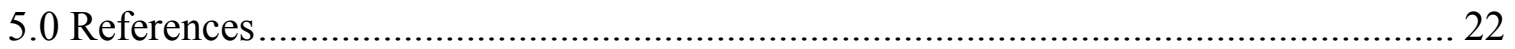




\section{LIST OF TABLES}

Table 3-1. Estimated Kilograms of Metal Compounds in Sludge Solids (SS) Received into the F Area Waste Tanks ..................................................................................... 7

Table 3-2. Estimated Kilograms of Metal Compounds in Sludge Solids (SS) Received

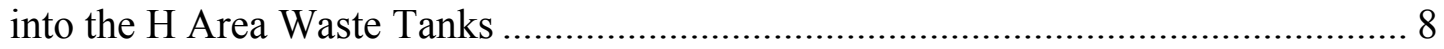

Table 3-3. Estimated Curies of Radionuclides in Sludge Solids (SS) Received into the F Area Waste Tanks (Curies have been adjusted for decay) ...................................... 9

Table 3-4. Estimated Curies of Radionuclides in Sludge Solids (SS) Received into the H Area Waste Tanks (Curies have been adjusted for decay) ..................................... 10

Table 3-5. Mineral Phases Identified in F and H Area Tank Farm Waste Solids........... 18 


\section{LIST OF ABBREVIATIONS}

$\begin{array}{ll}\text { CRC } & \text { Cesium removal column } \\ \text { DWPF } & \text { Defense Waste Processing Facility } \\ \text { FTF } & \text { F Tank Farm } \\ \text { HM } & \text { H-modified } \\ \text { HTF } & \text { H Tank Farm } \\ \text { RBOF } & \text { Receiving basin for offsite fuels } \\ \text { SRNL } & \text { Savannah River National Laboratory } \\ \text { SRS } & \text { Savannah River Site } \\ \text { SS } & \text { Sludge solids } \\ \text { TBP } & \text { Tributylphosphate } \\ \text { TTQAP } & \text { Task technical and quality assurance plan } \\ \text { XRD } & \text { X-ray diffraction }\end{array}$




\subsection{Introduction}

Approximately thirty-seven million gallons of liquid radioactive waste are currently stored in the F and H Area Tank Farms at the Savannah River Site. ${ }^{1}$ This waste was generated through decades of nuclear material processing activities targeting recovery of U-235, U-238, Np-237, Pu-238, Pu-239, and other key radioisotopes. The F Area Tank Farm (FTF) contains a total of twenty-two underground storage tanks, of which sixteen are still active and in use (the others are closed or in preparation for closure). In contrast, the H Area Tank Farm (HTF) contains a total of twenty-nine underground storage tanks, of which twenty-eight are still active and in use.

The composition of waste in the F and $\mathrm{H}$ Area tanks is a function of the processing activities, and as such, varies from tank to tank. Furthermore, the waste exists in three distinct forms - sludge, supernatant, and saltcake - and the proportions of the forms vary from tank to tank. As a consequence, both the distribution and quantities of waste constituents in any one storage tank can differ significantly from those of another. However, certain compositional trends can be projected for any given waste phase, given knowledge of the processing activities leading to generation of the waste.

Of particular importance from the tank closure perspective is the sludge phase, since it contains high concentrations of insoluble solids which typically drive the residual radioactivity that will ultimately remain in a tank after completion of bulk waste removal and cleaning. Utilizing knowledge of the processing differences impacting the composition of the sludge, expectations for compositional differences between $\mathrm{F}$ and $\mathrm{H}$ Area tank waste residues (after tank cleaning) can be developed. This includes differences related to distributions of radionuclides, stable constituents, and residue minerals. Such knowledge provides a basis for anticipating and projecting differences related to $\mathrm{F}$ and $\mathrm{H}$ Area environmental constituent fate and transport.

The goal of this document is to provide chemical information regarding $\mathrm{F}$ and $\mathrm{H}$ Area nuclear material processing and waste generation to make clear expectations regarding the relative abundances of stable and radiological constituents in the Tank Farm sludge solids. Specifically, this document will focus on the primary differences between sludge solids stored at the F and H Area Tank Farms. Such differences provide a basis anticipating differences between the $\mathrm{F}$ and $\mathrm{H}$ Area Tank Farm residual waste solids remaining after tank closure.

It should be noted that the chemical differences addressed in this document are based on expectations applicable to the average sludge solids received into FTF and HTF. Given that compositional waste variations are known to exist, and that the compositions of waste solids can be changed by mechanical and chemical cleaning operations, the differences identified in this document are not expected to be representative of any one individual FTF or HTF waste residue. As such, the results should only be used for anticipating general chemical trends between typical FTF and HTF waste, not specific chemical differences between waste residues in individual tanks. 
This document was generated in accordance with the protocols identified in Task Technical and Quality Assurance Plan (TTQAP) SRNL-STI-2012-00296 (TTQAP for HTF Performance Assessment Revision 1 Support).

\subsection{Objectives}

- Summarize F and H Area nuclear materials processing and waste characteristics

- Summarize primary FTF and HTF receipts

- Identify major differences between FTF and HTF waste

- Address mineral phases in FTF and HTF waste solids

- Address impacts of ancillary waste processing operations and chemical tank cleaning

- Address solid/liquid phase technetium partitioning

\subsection{Discussion}

\section{1 $\mathrm{F}$ and $\mathrm{H}$ Area Nuclear Materials Processing and Waste Characteristics}

Tank Farm waste is generated through two primary nuclear material recovery processes: the PUREX process and the H-modified process. ${ }^{2-6}$ A description of each process is given below.

\subsubsection{PUREX}

The PUREX process is used to recover uranium and plutonium from irradiated targets or fuel slugs containing a core of depleted or natural uranium (or slightly enriched uranium, in some limited cases). The acronym PUREX is taken from the words $\underline{\text { Plutonium }}$ URanium EXtraction.

In the PUREX process, aluminum cladding surrounding the targets is dissolved in a solution of sodium hydroxide, with sodium nitrate present to suppress hydrogen generation. The dejacketed targets/slugs are then dissolved in hot nitric acid solution, and the resulting digest solution is processed through a clarification process to remove the aluminum activation product silicon. The principal component of the digest solution is U-238, with smaller amounts of U-235 and various fission and activation products, including Pu-239 and Np-237.

The clarified digest solution is chemically adjusted and then processed through a series of cycles of liquid-liquid extraction, for the purpose of removing and purifying uranium and plutonium. The digest solution being fed to the first organic extraction cycle is referred to as the "head end" solution. The organic phase of the liquid-liquid extraction cycle is comprised of tributylphosphate (TBP) extractant mixed with n-paraffin diluent. The TBP concentration is typically 30 percent, by volume. 
After organic extraction, the resulting aqueous high activity waste stream (the stream from which uranium and plutonium were extracted) is processed through anion exchange, for recovery of neptunium and additional plutonium.

Following completion of the separations, the recovered nuclear materials are converted to forms conducive to subsequent use: a) $\mathrm{UO}_{3}$; b) $\mathrm{Pu}$ metal; and c) $\mathrm{NpO}_{2}$, fabricated into aluminum clad $\mathrm{NpO}_{2}-\mathrm{Al}$ targets for $\mathrm{Pu}-238$ production. In contrast, the aqueous waste streams are prepared for storage through concentration via evaporation, alkalization to $\mathrm{pH}$ $>13$ using $\mathrm{NaOH}$ (often referred to as neutralization), and subsequent transfer to the Tank Farm. It is the alkalization step that partitions most of the metals to the solid phase as metal hydroxides, oxides, or oxyhydroxides. The resulting insoluble metal solids are referred to as sludge solids. In contrast, the supernatant phase of the waste contains the soluble metal salts, primarily the sodium salts.

The most dominant metals in the waste stream are iron, aluminum, and uranium, and the second most dominant metals are sodium, manganese, nickel, calcium, and silicon. The principal sources of the metals are: a) for iron - use of ferrous sulfamate as a reducing agent in the organic extractions and the anion exchange separations; b) aluminum - from the cladding; c) uranium - residual target material not recovered during solvent extraction; d) sodium - from sodium hydroxide and sodium nitrate used for dissolution of the cladding, and from sodium hydroxide used to alkalize waste prior to storage; e) manganese - from manganese dioxide particles utilized to sorb/remove fission products from the "head end" solution, and from potassium permanganate used for equipment decontamination; f) nickel - from use as a bonding agent between the uranium target and the aluminum cladding; g) calcium - from plutonium scrap recovery; and h) silicon from activation of aluminum.

A vast array of radionuclides is present in the waste stream, including the uranium isotopes associated with the original target, as well as the fission and activation products generated through irradiation of the target, and the associated decay products. On an activity basis, the most dominant radionuclides are a function of the time elapsed since irradiation of the target, due to the range of radionuclide high-lives. For typical SRS Tank Farm waste (that is currently one to six decades old), the primary activity contributors are Sr-90 (and its short-lived decay product Y-90) and Cs-137 (and its short lived decay product $\mathrm{Ba}-137 \mathrm{~m}$ ). In contrast, on a mass basis, the most dominant radionuclide is $\mathrm{U}-238$, and the uranium isotope distribution follows that of the target, which is usually either depleted or natural uranium. In most cases, the plutonium isotope distribution is consistent with that of weapons grade material. The exceptions are cases where targets were irradiated for production of higher plutonium isotopes or other specialty isotopes. On an activity basis, the most dominant plutonium isotope at this point in time is typically $\mathrm{Pu}-238$.

The PUREX process was performed at both $\mathrm{F}$ and $\mathrm{H}$ Area Canyon/Recovery Facilities. As such, PUREX waste was received into both the F and H Area Tank Farms. 


\subsubsection{H-modified (HM)}

The H-modified (HM) process is similar to the PUREX process, but is tailored for recovering and separating uranium and neptunium from burned enriched uranium fuel (as opposed to irradiated depleted or natural uranium targets). The primary differences in the HM process are the manner that material is dissolved and the criticality safety requirements associated with the enriched uranium.

In the HM process, the aluminum cladding and the aluminum-uranium fuel alloy are dissolved simultaneously using a nitric acid solution catalyzed with mercuric nitrate. (This is considerably different than dissolution in the PUREX process, where aluminum cladding is dissolved in caustic solution, and target material is subsequently dissolved in uncatalyzed acid solution).

As in the PUREX process, the digest solution in the HM process is chemically adjusted and then processed through a series of cycles of liquid-liquid extraction, utilizing TBP extractant mixed with organic diluent. However, in the HM process, the TBP concentration used for uranium extraction is significantly lower, typically $7.5 \%$ (as opposed to $30 \%$ for PUREX). This lower extractant concentration assures a lower uranium concentration, which is necessary for nuclear safety control, due to the enriched U-235.

Following completion of the organic extractions, the purified uranium solution was sent offsite for conversion to uranium metal, and then recycled for use in fuel tubes. In contrast, the purified neptunium was concentrated by anion exchange, converted to oxide, fabricated into aluminum clad $\mathrm{NpO}_{2}-\mathrm{Al}$ alloy targets and returned to the reactors to produce $\mathrm{Pu}-238$.

Irradiated neptunium targets were dissolved using a nitric acid solution catalyzed with mercuric nitrate, just like the approach for dissolving burned uranium fuel. The Np-237 and $\mathrm{Pu}-238$ were then purified and separated by multiple cycles of anion exchange, and converted to oxides.

Although HM processing was primarily used for uranium fuel and neptunium targets, it was also used for processing a limited number of Th-232 targets irradiated for U-233 production, and for processing a limited number of other targets producing specialty isotopes.

Identical to the PUREX aqueous waste streams, the HM aqueous waste streams are prepared for storage through concentration via evaporation, alkalization to $\mathrm{pH}>13$ using $\mathrm{NaOH}$ (often referred to as neutralization), and subsequent transfer to the Tank Farm.

The dominant metals in the waste stream are very similar to those of the PUREX process, with a couple of exceptions. The iron and uranium contents of the HM waste are lower than those of the PUREX waste due to the lower relative quantities of uranium processed through HM because of the U-235 enrichment. The aluminum content of the HM waste 
is higher than that of the PUREX waste due to additional aluminum contributed by the UAl fuel alloy and the use of aluminum nitrate as a salting agent in the HM process. The mercury content of the HM waste is significantly higher than that of the PUREX waste due to its use as a dissolution catalyst in the HM process. The thorium content of Thorex waste is high. However, due to the relatively small number of Thorex campaigns, thorium was received into only a few of the Tank Farm tanks, and its concentration in those tanks is generally low, due to being consolidated with typical non-thorium-containing waste.

Like the PUREX waste, the HM waste contains a vast array of radionuclides, including those of the original fuel and targets, as well as those generated through fission, activation, and decay of the fission and activation products. Consistent with the PUREX waste, the primary activity contributors in typical Tank Farm HM waste (currently one to six decades old) are Sr-90 (and its short-lived decay product Y-90) and Cs-137 (and its short lived decay product $\mathrm{Ba}-137 \mathrm{~m}$ ). In contrast, on a mass basis, the radionuclides that dominate include U-235, U-238, Np-237, and Pu-239. In tanks that received thorium waste, Th-232 is another radionuclide that dominates from the mass perspective. On an activity basis, the current most dominant plutonium isotope by far is Pu-238.

The HM process was performed solely at the H Area Canyon/Recovery Facility. As such, HM waste was received solely into the HTF.

\subsection{F and H Area Tank Farm Waste Receipts}

Tank Farm waste receipt records provide a technical basis for projecting waste compositions as a function of the corresponding nuclear processing activities. Such records have been compiled, summarized, and coupled with process knowledge to estimate the quantities of chemical and radiological constituents comprising the sludge solids. $^{7-11}$ This information has been captured in SRR's Sludge 1.5 electronic spreadsheets, ${ }^{12}$ and provides compositional breakdowns of the sludge solids of each waste receipt as a function of the receiving waste tank and the waste receipt date.

Projected quantities of thirty-six metal compounds and thirty-seven radionuclides in each waste receipt are given in the Sludge 1.5 spreadsheets. The metal compounds that are addressed include: silver hydroxide $(\mathrm{AgOH})$, aluminum hydroxide $\left(\mathrm{Al}(\mathrm{OH})_{3}\right)$, barium sulfate $\left(\mathrm{BaSO}_{4}\right)$, calcium phosphate $\left(\mathrm{Ca}_{3}\left(\mathrm{PO}_{4}\right)_{2}\right)$, calcium oxalate $\left(\mathrm{CaC}_{2} \mathrm{O}_{4}\right)$, calcium carbonate $\left(\mathrm{CaCO}_{3}\right)$, calcium fluoride $\left(\mathrm{CaF}_{2}\right)$, calcium sulfate $\left(\mathrm{CaSO}_{4}\right)$, cerium hydroxide $\left(\mathrm{Ce}(\mathrm{OH})_{3}\right)$, cobalt hydroxide $\left(\mathrm{Co}(\mathrm{OH})_{3}\right)$, chromium hydroxide $\left(\mathrm{Cr}(\mathrm{OH})_{3}\right)$, copper hydroxide $\left(\mathrm{Cu}(\mathrm{OH})_{2}\right)$, iron hydroxide $\left(\mathrm{Fe}(\mathrm{OH})_{3}\right)$, mercuric oxide $(\mathrm{HgO})$, potassium nitrate $\left(\mathrm{KNO}_{3}\right)$, lanthanum hydroxide $\left(\mathrm{La}(\mathrm{OH})_{3}\right)$, magnesium hydroxide $\left(\mathrm{Mg}(\mathrm{OH})_{2}\right)$, manganese dioxide $\left(\mathrm{MnO}_{2}\right)$, sodium sulfate $\left(\mathrm{Na}_{2} \mathrm{SO}_{4}\right)$, sodium phosphate $\left(\mathrm{Na}_{3} \mathrm{PO}_{4}\right)$, sodium chloride $(\mathrm{NaCl})$, sodium fluoride $(\mathrm{NaF})$, sodium iodide $(\mathrm{NaI})$, sodium nitrate $\left(\mathrm{NaNO}_{3}\right)$, sodium hydroxide $(\mathrm{NaOH})$, nickel hydroxide $\left(\mathrm{Ni}(\mathrm{OH})_{2}\right)$, lead carbonate $\left(\mathrm{PbCO}_{3}\right)$, lead sulfate $\left(\mathrm{PbSO}_{4}\right)$, praseodymium hydroxide $\left(\mathrm{Pr}(\mathrm{OH})_{3}\right)$, ruthenium dioxide $\left(\mathrm{RuO}_{2}\right)$, silicon dioxide $\left(\mathrm{SiO}_{2}\right)$, strontium carbonate $\left(\mathrm{SrCO}_{3}\right)$, thorium dioxide $\left(\mathrm{ThO}_{2}\right)$, 
uranyl hydroxide $\left(\mathrm{UO}_{2}(\mathrm{OH})_{2}\right)$, zinc hydroxide $\left(\mathrm{Zn}(\mathrm{OH})_{2}\right)$, and zirconyl hydroxide $\left(\mathrm{ZrO}(\mathrm{OH})_{2}\right)$.

The radionuclides that are addressed include: carbon-14 (C-14), cobalt-60 (Co-60), nickel-59 (Ni-59), selenium-79 (Se-79), strontium-90 (Sr-90), yttrium-90 (Y-90), technetium-99 (Tc-99), ruthenium-106 (Ru-106), rhodium-106 (Rh-106), antimony-125 (Sb-125), tin-126 (Sn-126), iodine-129 (I-129), cesium-134 (Cs-134), cesium-135 (Cs135), cesium-137 (Cs-137), barium-137 metastable (Ba-137m), cerium-144 (Ce-144), praseodymium-144 (Pr-144), promethium-147 (Pm-147), europium-154 (Eu-154), thorium-232 (Th-232), uranium-232 (U-232), uranium-233 (U-233), uranium-234 (U234), uranium-235 (U-235), uranium-236 (U-236), uranium-238 (U-238), neptunium-237 (Np-237), plutonium-238 (Pu-238), plutonium-239 (Pu-239), plutonium-240 (Pu-240), plutonium-241 ( $\mathrm{Pu}-241)$, plutonium-242 (Pu-242), americium-241 (Am-241), americium242 metastable (Am-242m), curium-244 (Cm-244), and curium-245 (Cm-245).

Estimated quantities of the metal compounds in each waste receipt are presented in Sludge 1.5 spreadsheets in terms of mass, in units of kilograms $(\mathrm{kg})$. In contrast, the estimated quantities of the radionuclides are given in terms of activity, in units of Curies (Ci). The radionuclide activities have been adjusted for decay that has occurred from the time the waste was received. For each tank that has received waste from the canyons, Sludge 1.5 identifies the history of the waste receipts, specifying the type of waste and composition of sludge solids associated with each waste receipt. This history identifies fifteen F Area tanks that received PUREX (P) waste from F-Canyon: Tanks 1-8, 17-19, 26, 33, 34, and 47; and seventeen H Area tanks that received PUREX and/or HM waste from H-Canyon: Tanks 9-16, 21, 22, 30, 32, 35, 36, 39, 41, and 43. Tank 9 received PUREX waste only, Tanks 10-14 received a combination of PUREX and HM waste, and the remaining $\mathrm{H}$ Area Canyon receipt tanks received HM waste only. Although the majority of $\mathrm{F}$ and $\mathrm{H}$ Area waste receipts were high metal content sludge waste, some of the receipts were liquid canyon flushes and/or ancillary process discards with negligible sludge/metal content.

The total quantity of each metal compound and each radionuclide received into a tank was calculated by summing the contributions of the individual waste receipts. The results are given in Tables 3-1 to 3-4, with: a) Table 3-1 identifying the total estimated masses of metal compounds received into each F Area Canyon receipt tank; b) Table 3-2 identifying the total estimated masses of metal compounds received into each $\mathrm{H}$ Area Canyon receipt tank; c) Table 3-3 identifying the total estimated activities of radionuclides received into each F Area Canyon receipt tank; and d) Table 3-4 identifying the total estimated activities of radionuclides received into each $\mathrm{H}$ Area Canyon receipt tank.

Also included in Tables 3-1 and 3-2 are: a) the total mass of each metal compound received into the Tank Farm (second to last row); b) the total mass of sludge solids received into the Tank Farm (last column); and c) the percentage of the Tank Farm sludge mass that is contributed by each metal compound (last row). 
Table 3-1. Estimated Kilograms of Metal Compounds in Sludge Solids (SS) Received into the F Area Waste Tanks

\begin{tabular}{|c|c|c|c|c|c|c|c|c|c|c|c|c|c|c|c|c|c|c|c|}
\hline Tank \# & Receipts & 동 & 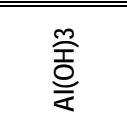 & $\begin{array}{l}\text { ర్ } \\
\text { J్ల్ల }\end{array}$ & 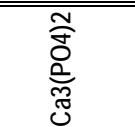 & $\begin{array}{l}\text { ర్ల్ } \\
\text { ర్ల }\end{array}$ & 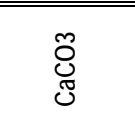 & $\begin{array}{l}\text { ్ㅀ } \\
\widetilde{J}\end{array}$ & $\begin{array}{l}\text { ఫ్ర్ } \\
\text { శ్ }\end{array}$ & 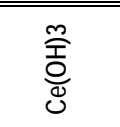 & $\frac{\text { }}{\tilde{J}}$ & 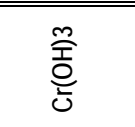 & 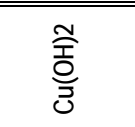 & 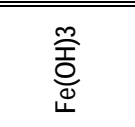 & ㅇㅗㅗ & $\sum_{\frac{1}{2}}^{\infty}$ & $\underset{\widetilde{J}}{\stackrel{m}{\underline{T}}}$ & $\frac{\widetilde{N}}{\frac{0}{\bar{D}}}$ & $\tilde{\tilde{c}}$ \\
\hline $1 \mathrm{~F}$ & $P$ & $6.9 \mathrm{E}+01$ & $5.7 \mathrm{E}+03$ & $1.9 \mathrm{E}+02$ & $9.0 \mathrm{E}+01$ & $0.0 \mathrm{E}+00$ & $1.3 E+03$ & $5.9 \mathrm{E}+01$ & $1.0 \mathrm{E}+02$ & $1.0 \mathrm{E}+02$ & $4.0 \mathrm{E}+00$ & $1.9 \mathrm{E}+02$ & $6.5 \mathrm{E}+01$ & $1.6 \mathrm{E}+04$ & $5.5 \mathrm{E}+01$ & $1.8 \mathrm{E}+02$ & $5.4 \mathrm{E}+01$ & $1.4 \mathrm{E}+02$ & $8.2 \mathrm{E}+03$ \\
\hline $2 \mathrm{~F}$ & $\mathrm{P}$ & $3.2 \mathrm{E}+01$ & $6.4 \mathrm{E}+02$ & $5.9 \mathrm{E}+01$ & $3.0 \mathrm{E}+01$ & $0.0 \mathrm{E}+00$ & $5.6 \mathrm{E}+02$ & $2.9 \mathrm{E}+01$ & $5.4 \mathrm{E}+01$ & $4.0 \mathrm{E}+01$ & $2.1 \mathrm{E}+00$ & $6.4 \mathrm{E}+01$ & $2.6 \mathrm{E}+01$ & $5.9 \mathrm{E}+03$ & $1.6 \mathrm{E}+01$ & $8.1 \mathrm{E}+01$ & $2.2 \mathrm{E}+01$ & $4.1 \mathrm{E}+01$ & $4.9 \mathrm{E}+03$ \\
\hline $3 \mathrm{~F}$ & $P$ & $7.1 \mathrm{E}+01$ & $1.6 \mathrm{E}+03$ & $\frac{1.3 \mathrm{E}+02}{1}$ & $6.0 \mathrm{E}+01$ & $0.0 \mathrm{E}+00$ & $1.2 \mathrm{E}+03$ & $6.4 \mathrm{E}+01$ & $\begin{array}{l}1.4 \mathrm{~L}+\mathrm{L} \\
.2 \mathrm{E}+02\end{array}$ & $8.8 \mathrm{E}+01$ & $4.6 \mathrm{E}+00$ & $\begin{array}{l}1.4 \mathrm{~L}+02 \\
\end{array}$ & $5.7 \mathrm{E}+01$ & $1.3 \mathrm{E}+04$ & $\frac{1.0 \mathrm{~L}}{3.5 \mathrm{E}+01}$ & $\frac{0.1 \mathrm{~L}}{1.8 \mathrm{E}+02}$ & $4.9 \mathrm{E}+01$ & $9.2 \mathrm{E}+01$ & $1.3 \mathrm{E}+04$ \\
\hline $4 \mathrm{~F}$ & $P$ & $4.5 \mathrm{E}+01$ & $6.7 \mathrm{E}+03$ & $3.3 \mathrm{E}+02$ & $1.4 \mathrm{E}+02$ & $0.0 \mathrm{E}+00$ & $1.3 \mathrm{E}+03$ & $3.1 \mathrm{E}+01$ & $3.1 \mathrm{E}+01$ & $1.2 \mathrm{E}+02$ & $1.2 \mathrm{E}+00$ & $2.9 \mathrm{E}+02$ & $7.2 \mathrm{E}+01$ & $2.1 \mathrm{E}+04$ & $9.9 \mathrm{E}+01$ & $1.4 \mathrm{E}+02$ & $5.6 \mathrm{E}+01$ & $2.4 \mathrm{E}+02$ & $6.5 \mathrm{E}+03$ \\
\hline $5 F$ & $P$ & $1.1 \mathrm{E}+02$ & $3.4 \mathrm{E}+03$ & $2.8 \mathrm{E}+02$ & $1.3 \mathrm{E}+02$ & $0.0 \mathrm{E}+00$ & $2.0 \mathrm{E}+03$ & $9.2 \mathrm{E}+01$ & $1.6 \mathrm{E}+02$ & $1.5 \mathrm{E}+02$ & $6.2 \mathrm{E}+00$ & $2.8 \mathrm{E}+02$ & $9.8 \mathrm{E}+01$ & $2.4 \mathrm{E}+04$ & $8.0 \mathrm{E}+01$ & $2.8 \mathrm{E}+02$ & $8.2 \mathrm{E}+01$ & $2.0 \mathrm{E}+02$ & $5.5 \mathrm{E}+03$ \\
\hline $6 \mathrm{~F}$ & $\mathrm{P}$ & $1.5 \mathrm{E}+01$ & $6.3 \mathrm{E}+03$ & $1.7 \mathrm{E}+02$ & $6.8 \mathrm{E}+01$ & $0.0 \mathrm{E}+00$ & $5.8 \mathrm{E}+02$ & $8.2 \mathrm{E}+00$ & $0.0 \mathrm{E}+00$ & $5.4 \mathrm{E}+01$ & $0.0 \mathrm{E}+00$ & $1.4 \mathrm{E}+02$ & $3.2 \mathrm{E}+01$ & $1.0 \mathrm{E}+04$ & $5.0 \mathrm{E}+01$ & $5.2 \mathrm{E}+01$ & $2.5 \mathrm{E}+01$ & $1.2 \mathrm{E}+02$ & $2.4 \mathrm{E}+03$ \\
\hline $7 \mathrm{~F}$ & $P$ & $1.2 \mathrm{E}+03$ & $8.9 \mathrm{E}+04$ & $1.2 \mathrm{E}+03$ & $7.2 \mathrm{E}+02$ & $0.0 \mathrm{E}+00$ & $1.9 \mathrm{E}+04$ & $1.1 \mathrm{E}+03$ & $2.2 \mathrm{E}+03$ & $1.2 \mathrm{E}+03$ & $8.5 \mathrm{E}+01$ & $1.6 \mathrm{E}+03$ & $8.3 \mathrm{E}+02$ & $1.7 \mathrm{E}+05$ & $2.9 \mathrm{E}+02$ & $3.0 \mathrm{E}+03$ & $7.3 \mathrm{E}+02$ & $8.4 \mathrm{E}+02$ & $3.1 \mathrm{E}+04$ \\
\hline $8 \mathrm{~F}$ & $\mathrm{P}$ & $4.5 \mathrm{E}+02$ & $3.8 \mathrm{E}+04$ & $6.3 \mathrm{E}+02$ & $3.4 \mathrm{E}+02$ & $0.0 \mathrm{E}+00$ & $7.3 \mathrm{E}+03$ & $4.1 \mathrm{E}+02$ & $7.8 \mathrm{E}+02$ & $5.0 \mathrm{E}+02$ & $3.0 \mathrm{E}+01$ & $7.4 \mathrm{E}+02$ & $3.3 \mathrm{E}+02$ & $7.2 \mathrm{E}+04$ & $1.6 \mathrm{E}+02$ & $1.1 \mathrm{E}+03$ & $2.9 \mathrm{E}+02$ & $4.4 \mathrm{E}+02$ & $6.7 \mathrm{E}+03$ \\
\hline $17 \mathrm{~F}$ & $P$ & $1.7 \mathrm{E}+03$ & $7.3 \mathrm{E}+04$ & $1.5 \mathrm{E}+03$ & $9.2 \mathrm{E}+02$ & $0.0 \mathrm{E}+00$ & $2.6 \mathrm{E}+04$ & $1.6 \mathrm{E}+03$ & $3.2 \mathrm{E}+03$ & $1.7 \mathrm{E}+03$ & $1.2 \mathrm{E}+02$ & $2.1 \mathrm{E}+03$ & $1.1 \mathrm{E}+03$ & $2.3 \mathrm{E}+05$ & $3.3 \mathrm{E}+02$ & $4.2 \mathrm{E}+03$ & $1.0 \mathrm{E}+03$ & $9.9 \mathrm{E}+02$ & $8.9 \mathrm{E}+03$ \\
\hline $18 \mathrm{~F}$ & $\mathrm{P}$ & $1.9 \mathrm{E}+03$ & $9.4 \mathrm{E}+04$ & $1.6 \mathrm{E}+03$ & $1.0 \mathrm{E}+03$ & $0.0 \mathrm{E}+00$ & $2.8 \mathrm{E}+04$ & $1.7 \mathrm{E}+03$ & $3.5 \mathrm{E}+03$ & $1.8 \mathrm{E}+03$ & $1.3 \mathrm{E}+02$ & $2.2 \mathrm{E}+03$ & $1.2 \mathrm{E}+03$ & $2.5 \mathrm{E}+05$ & $3.6 \mathrm{E}+02$ & $4.5 \mathrm{E}+03$ & $1.1 \mathrm{E}+03$ & $1.1 \mathrm{E}+03$ & $9.6 \mathrm{E}+03$ \\
\hline $19 \mathrm{~F}$ & $\mathrm{P}$ & $1.1 \mathrm{E}+01$ & $4.6 \mathrm{E}+02$ & $9.6 \mathrm{E}+00$ & $6.0 \mathrm{E}+00$ & $0.0 \mathrm{E}+00$ & $1.7 \mathrm{E}+02$ & $1.1 \mathrm{E}+01$ & $2.1 \mathrm{E}+01$ & $1.1 \mathrm{E}+01$ & $8.0 \mathrm{E}-01$ & $1.4 \mathrm{E}+01$ & $7.4 \mathrm{E}+00$ & $1.5 \mathrm{E}+03$ & $2.2 \mathrm{E}+00$ & $2.7 \mathrm{E}+01$ & $6.6 \mathrm{E}+00$ & $6.5 \mathrm{E}+00$ & $5.7 \mathrm{E}+01$ \\
\hline $26 \mathrm{~F}$ & $\mathrm{P}$ & $5.0 \mathrm{E}+02$ & $4.4 \mathrm{E}+04$ & $4.3 \mathrm{E}+02$ & $2.7 \mathrm{E}+02$ & $0.0 \mathrm{E}+00$ & $7.6 \mathrm{E}+03$ & $4.7 \mathrm{E}+02$ & $9.3 \mathrm{E}+02$ & $4.9 \mathrm{E}+02$ & $3.6 \mathrm{E}+01$ & $6.1 \mathrm{E}+02$ & $3.3 \mathrm{E}+02$ & $6.7 \mathrm{E}+04$ & $9.7 \mathrm{E}+01$ & $1.2 \mathrm{E}+03$ & $3.0 \mathrm{E}+02$ & $2.9 \mathrm{E}+02$ & $5.3 \mathrm{E}+03$ \\
\hline $33 \mathrm{~F}$ & $\mathrm{P}$ & $4.0 \mathrm{E}+02$ & $1.2 \mathrm{E}+04$ & $7.9 \mathrm{E}+02$ & $3.9 \mathrm{E}+02$ & $0.0 \mathrm{E}+00$ & $7.0 \mathrm{E}+03$ & $3.6 \mathrm{E}+02$ & $6.6 \mathrm{E}+02$ & $5.1 \mathrm{E}+02$ & $2.5 \mathrm{E}+01$ & $8.4 \mathrm{E}+02$ & $3.3 \mathrm{E}+02$ & $7.7 \mathrm{E}+04$ & $2.1 \mathrm{E}+02$ & $1.0 \mathrm{E}+03$ & $2.8 \mathrm{E}+02$ & $5.6 \mathrm{E}+02$ & $7.6 \mathrm{E}+02$ \\
\hline $34 \mathrm{~F}$ & $\mathrm{P}$ & $3.3 \mathrm{E}+01$ & $6.4 \mathrm{E}+03$ & $3.7 \mathrm{E}+02$ & $1.5 \mathrm{E}+02$ & $0.0 \mathrm{E}+00$ & $1.3 \mathrm{E}+03$ & $1.8 \mathrm{E}+01$ & $0.0 \mathrm{E}+00$ & $1.2 \mathrm{E}+02$ & $0.0 \mathrm{E}+00$ & $3.2 \mathrm{E}+02$ & $7.1 \mathrm{E}+01$ & $2.2 \mathrm{E}+04$ & $1.1 \mathrm{E}+02$ & $1.1 \mathrm{E}+02$ & $5.4 \mathrm{E}+01$ & $2.7 \mathrm{E}+02$ & $9.3 \mathrm{E}+00$ \\
\hline $47 \mathrm{~F}$ & $\mathrm{P}$ & $4.6 \mathrm{E}+02$ & $3.7 \mathrm{E}+04$ & $4.0 \mathrm{E}+02$ & $2.5 \mathrm{E}+02$ & $0.0 \mathrm{E}+00$ & $7.0 \mathrm{E}+03$ & $4.3 \mathrm{E}+02$ & $8.6 \mathrm{E}+02$ & $4.6 \mathrm{E}+02$ & $3.3 \mathrm{E}+01$ & $5.6 \mathrm{E}+02$ & $3.1 \mathrm{E}+02$ & $6.2 \mathrm{E}+04$ & $8.9 \mathrm{E}+01$ & $1.1 \mathrm{E}+03$ & $2.7 \mathrm{E}+02$ & $2.7 \mathrm{E}+02$ & $4.5 \mathrm{E}+03$ \\
\hline Total k & & $7.0 \mathrm{E}+03$ & $4.2 \mathrm{E}+05$ & $8.1 \mathrm{E}+03$ & $4.6 \mathrm{E}+03$ & $0.0 \mathrm{E}+00$ & $1.1 \mathrm{E}+05$ & $6.5 \mathrm{E}+03$ & $1.3 \mathrm{E}+04$ & $7.4 \mathrm{E}+03$ & $4.9 \mathrm{E}+02$ & $1.0 \mathrm{E}+04$ & $4.9 \mathrm{E}+03$ & $1.0 \mathrm{E}+06$ & $2.0 \mathrm{E}+03$ & $1.7 \mathrm{E}+04$ & $4.3 \mathrm{E}+03$ & $5.6 \mathrm{E}+03$ & $1.1 \mathrm{E}+05$ \\
\hline$\%$ of SS & & $2.9 \mathrm{E}-01$ & $1.7 \mathrm{E}+01$ & $3.4 \mathrm{E}-01$ & $1.9 \mathrm{E}-01$ & $0.0 \mathrm{E}+00$ & $4.6 \mathrm{E}+00$ & $2.7 \mathrm{E}-01$ & $5.2 \mathrm{E}-01$ & $3.1 \mathrm{E}-01$ & $2.0 \mathrm{E}-02$ & $4.2 \mathrm{E}-01$ & $2.0 \mathrm{E}-01$ & $4.3 \mathrm{E}+01$ & $8.3 \mathrm{E}-02$ & $7.1 \mathrm{E}-01$ & $1.8 \mathrm{E}-01$ & $2.3 \mathrm{E}-01$ & $4.5 \mathrm{E}+00$ \\
\hline
\end{tabular}

\begin{tabular}{|c|c|c|c|c|c|c|c|c|c|c|c|c|c|c|c|c|c|c|c|}
\hline Tank \# & 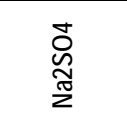 & $\begin{array}{l}\text { ठ } \\
\text { o. } \\
\frac{\pi}{2}\end{array}$ & $\begin{array}{l}\overline{\bar{\sigma}} \\
\tilde{z}\end{array}$ & 嵌 & $\frac{\bar{\pi}}{2}$ & 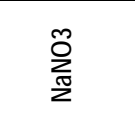 & 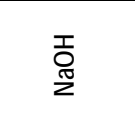 & $\frac{\text { 颃 }}{\frac{2}{z}}$ & O్ర & 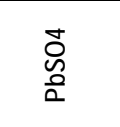 & 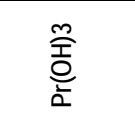 & ָั & 음 & 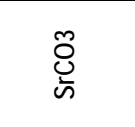 & 옽 & 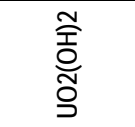 & $\frac{\widetilde{N}}{\frac{\partial}{\Sigma}}$ & $\begin{array}{l}\tilde{N} \\
\frac{\tilde{O}}{0} \\
\frac{\partial}{N}\end{array}$ & $\begin{array}{c}\text { Total } \\
\text { SS } \\
\text { Mass, } \\
\text { kg }\end{array}$ \\
\hline $1 \mathrm{~F}$ & $0.0 \mathrm{E}+00$ & $.0 \mathrm{E}+00$ & 95 & $.0 \mathrm{E}+00$ & 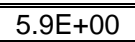 & $9 \mathrm{E}+02$ & $1.6 \mathrm{E}+03$ & $5.2 \mathrm{E}+03$ & $8.9 \mathrm{E}+01$ & $7.8 \mathrm{E}+01$ & $5.4 \mathrm{E}+01$ & $1.7 \mathrm{E}+02$ & 4.0LTUL & $7 \bar{E}+01$ & $\overline{00}$ & $7.3 \mathrm{E}+03$ & $1.2 \mathrm{E}+02$ & $2.3 \mathrm{E}+02$ & $4.9 \mathrm{E}+04$ \\
\hline $2 F$ & $E+00$ & $E+00$ & $\mathrm{E}+02$ & $\mathrm{E}+00$ & $E+00$ & $\mathrm{E}+02$ & $9 \mathrm{E}+02$ & $1.1 \mathrm{E}+03$ & $\mathrm{~F}+01$ & $255+01$ & $2.2 \mathrm{E}+01$ & $7.1 \mathrm{E}+01$ & $1.9 \mathrm{E}+02$ & $1 \mathrm{E}+01$ & & $7.7 \mathrm{E}+02$ & $.8 \mathrm{E}+01$ & $9.0 \mathrm{E}+01$ & +04 \\
\hline $3 \mathrm{~F}$ & & & $E+02$ & 0 & & $5+0 ?$ & $25+03$ & $\mathrm{E}+03$ & & & $9 \mathrm{9}+01$ & $16 \mathrm{E}+0$ & & & & $2.4 \mathrm{E}+03$ & $1.1 \mathrm{E}+02$ & $2.0 \mathrm{E}+02$ & \\
\hline $4 \mathrm{~F}$ & +00 & +00 & $\mathrm{E}+02$ & $0.0 \mathrm{E}+00$ & +00 & $\mathrm{E}+02$ & $2 \mathrm{E}+03$ & $1.1 \mathrm{E}+04$ & $9 \mathrm{E}+02$ & $5.9 E+01$ & $6 \mathrm{E}+01$ & 1.8 & 02 & 1.1 & 0.0 & $1.3 \mathrm{E}+04$ & $1.1 \mathrm{E}+02$ & $2.7 \mathrm{E}+02$ & +04 \\
\hline $5 \mathrm{~F}$ & & & & & & & & & & & & & & & & & & & \\
\hline $6 \mathrm{~F}$ & & & & & & & & $7.1 \mathrm{E}+03$ & & $2.2 \mathrm{E}+01$ & $2.4 \mathrm{E}+0$ & & & & & $9.5 \mathrm{E}+03$ & $4.9 \mathrm{E}+01$ & $1.2 \mathrm{E}+02$ & +04 \\
\hline $7 \mathrm{~F}$ & & & & & & 03 & & & & $1.3 \mathrm{E}+03$ & $7.3 \mathrm{E}+02$ & & & & & $3.5 \mathrm{E}+04$ & $1.6 \mathrm{E}+03$ & $2.8 \mathrm{E}+03$ & $E+05$ \\
\hline $8 \mathrm{~F}$ & & & 2. & & & 2 & $7.2 \mathrm{E}$ & $6.9 \mathrm{E}+03$ & & $4.8 \mathrm{E}+02$ & & & & & & $2.6 \mathrm{E}+04$ & $6.3 \mathrm{E}+02$ & $1.1 \mathrm{E}+03$ & +05 \\
\hline $17 \mathrm{~F}$ & & & & & & & & & & & & & & & & & & & \\
\hline $18 \mathrm{~F}$ & & & & & & & 2. & & & & & & & & & $2.0 \mathrm{E}+04$ & $2.4 \mathrm{E}+03$ & $4.2 \mathrm{E}+03$ & -05 \\
\hline $19 \mathrm{~F}$ & & & $6.5 \mathrm{E}$ & & & & & & & & & & & & & 7.0 & $1.5 \mathrm{E}+01$ & $2.5 \mathrm{E}+01$ & \\
\hline $26 \mathrm{~F}$ & $E^{+}$ & & $2.9 \mathrm{E}+03$ & & $4 \mathrm{E}$ & $2.2 \mathrm{E}$ & $6.6 \mathrm{E}$ & & & $5.3 \mathrm{E}$ & & & 2.6 & & & 1.2 & $6.6 \mathrm{E}$ & $1.1 \mathrm{E}+03$ & $1.6 \mathrm{E}+05$ \\
\hline $33 \mathrm{~F}$ & & & & & & & & & & & & & & & & & & & \\
\hline $34 \mathrm{~F}$ & & & $9.5 \mathrm{E}$ & & & & & & & & & & & & & 3.1 & $1.1 \mathrm{E}+02$ & $2.7 \mathrm{E}+02$ & \\
\hline $47 \mathrm{~F}$ & $0.0 \mathrm{E}+00$ & $0.0 \mathrm{E}+00$ & $2.7 \mathrm{E}+03$ & $0.0 \mathrm{E}+00$ & $4.9 \mathrm{E}+01$ & $2.0 \mathrm{E}+03$ & $6.0 \mathrm{E}+03$ & $0.0 \mathrm{E}+00$ & $0.0 \mathrm{E}+00$ & $4.9 \mathrm{E}+02$ & $2.7 \mathrm{E}+02$ & $8.8 \mathrm{E}+02$ & $2.4 \mathrm{E}+03$ & $1.5 \mathrm{E}+02$ & $0.0 \mathrm{E}+00$ & $5.6 \mathrm{E}+03$ & $6.1 \mathrm{E}+02$ & $1.0 \mathrm{E}+03$ & $1.4 \mathrm{E}+05$ \\
\hline Total k & $\overline{0}$ & 0. & & & & & & & & & & & & & & & & & \\
\hline$\%$ of & $0.0 \mathrm{E}+00$ & $0.0 \mathrm{E}+00$ & $1.7 \mathrm{E}+00$ & $0.0 \mathrm{E}+00$ & $3.0 \mathrm{E}-02$ & $1.4 \mathrm{E}+00$ & $4.3 \mathrm{E}+00$ & $2.6 \mathrm{E}+00$ & 5. & $3.1 \mathrm{E}-01$ & $1.8 \mathrm{E}-01$ & $5.8 \mathrm{E}-01$ & $1.6 \mathrm{E}+00$ & $1.2 \mathrm{E}-01$ & $0.0 \mathrm{E}+00$ & $1.3 \mathrm{E}+01$ & $4.0 \mathrm{E}-01$ & $7.0 \mathrm{E}-01$ & $1.0 \mathrm{E}+02$ \\
\hline
\end{tabular}


Table 3-2. Estimated Kilograms of Metal Compounds in Sludge Solids (SS) Received into the H Area Waste Tanks

\begin{tabular}{|c|c|c|c|c|c|c|c|c|c|c|c|c|c|c|c|c|c|c|c|}
\hline Tank \# & Receipts & 징 & $\frac{\frac{\mathrm{m}}{\mathrm{\sigma}}}{\frac{\mathrm{a}}{\alpha}}$ & $\begin{array}{l}\text { ర్ } \\
\text { ల్ల } \\
\text { D. }\end{array}$ & 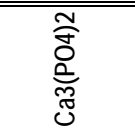 & $\begin{array}{l}\text { ర్ల్ర } \\
\text { రٓ }\end{array}$ & $\begin{array}{l}\text { ల్ల } \\
\text { ల్ల }\end{array}$ & $\begin{array}{l}\text { 芯 } \\
\widetilde{J}\end{array}$ & $\begin{array}{l}\text { ర్ } \\
\text { ర్ల }\end{array}$ & 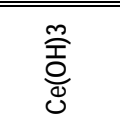 & $\begin{array}{l}\frac{m}{\widetilde{J}} \\
\frac{0}{\delta}\end{array}$ & 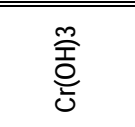 & 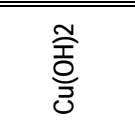 & 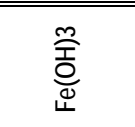 & 옴 & $\sum_{\underline{x}}^{0}$ & 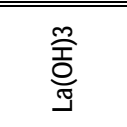 & $\frac{\frac{N}{\bar{T}}}{\frac{D}{2}}$ & 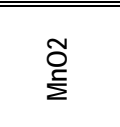 \\
\hline $9 \mathrm{H}$ & $P$ & $1 E+01$ & $1 \mathrm{E}+02$ & $5.6 \mathrm{E}+01$ & $2.8 \mathrm{E}+01$ & $0.0 \mathrm{E}+00$ & $5.3 \mathrm{E}+02$ & $2.7 \mathrm{E}+01$ & $1 \mathrm{E}+01$ & $3.8 \mathrm{E}+01$ & $2.0 \mathrm{E}+00$ & $6.1 \mathrm{E}+01$ & $2.5 \mathrm{E}+01$ & $5.6 \mathrm{E}+03$ & $.5 \mathrm{E}+01$ & $7 \mathrm{E}+01$ & $2.1 \mathrm{E}+01$ & $3.9 \mathrm{E}+01$ & $4.5 \mathrm{E}+03$ \\
\hline $10 \mathrm{H}$ & and $\mathrm{HM}$ & +01 & $\mathrm{E}+03$ & +01 & $8 \mathrm{E}+01$ & $\mathrm{DE}+00$ & $9.1 \mathrm{E}+02$ & $7 \mathrm{E}+01$ & $8 \mathrm{E}+01$ & $6.5 \mathrm{E}+01$ & $3.4 \mathrm{E}+00$ & $1.0 \mathrm{E}+02$ & $4.2 \mathrm{E}+01$ & $9.7 \mathrm{E}+03$ & $2.6 \mathrm{E}+01$ & $1.3 \mathrm{E}+02$ & $3.6 \mathrm{E}+01$ & $6.8 \mathrm{E}+01$ & $1.2 \mathrm{E}+04$ \\
\hline $11 \mathrm{H}$ & and $\mathrm{HM}$ & 0 & $1.1 \mathrm{E}+05$ & $1 \mathrm{E}+02$ & $2.2 \mathrm{E}+02$ & $2 \mathrm{E}+03$ & $1.8 \mathrm{E}+03$ & $\mathrm{EE}+00$ & 00 & $6.0 \mathrm{E}+02$ & $.0 \mathrm{E}+00$ & $6.1 \mathrm{E}+02$ & $1.3 \mathrm{E}+02$ & $4.3 \mathrm{E}+04$ & $8.1 \mathrm{E}+03$ & & $2.5 \mathrm{E}+02$ & $8.9 \mathrm{E}+02$ & $7.4 \mathrm{E}+03$ \\
\hline $12 \mathrm{H}$ & $\mathrm{P}$ and $\mathrm{HM}$ & $5.9 \mathrm{E}+01$ & $7.6 \mathrm{E}+04$ & $8 \mathrm{E}+02$ & $2.4 \mathrm{E}+02$ & $4.4 \mathrm{E}+03$ & $1.0 \mathrm{E}+03$ & $5.3 \mathrm{E}+01$ & $9.9 \mathrm{E}+01$ & $1.8 \mathrm{E}+02$ & $3.8 \mathrm{E}+00$ & $5.8 \mathrm{E}+02$ & $1.5 \mathrm{E}+02$ & $3.2 \mathrm{E}+04$ & $6.1 \mathrm{E}+03$ & $6.0 \mathrm{E}$ & $1.3 \mathrm{E}+02$ & $6.2 \mathrm{E}+02$ & $2.3 \mathrm{E}+04$ \\
\hline $13 \mathrm{H}$ & $\mathrm{D}$ and & مा & $3.1 \mathrm{E}+04$ & & $2.5 \mathrm{E}+01$ & $1 \mathrm{~F}+0 ?$ & $1-5$ & $0.0 \mathrm{E}$ & & $4.3 \mathrm{E}+03$ & $0.0 \mathrm{E}+00$ & $6.2 \mathrm{E}+02$ & $1.6 \mathrm{E}+02$ & $1.7 \mathrm{E}+05$ & $8.6 \mathrm{E}$ & & $1.3 \mathrm{E}+03$ & & \\
\hline $14 \mathrm{H}$ & $\mathrm{dH}$ & E- & $4.4 \mathrm{E}+03$ & & $4.2 \mathrm{E}+01$ & $3 \mathrm{E}+02$ & $8 \mathrm{E}+02$ & 0 & & $7.8 \mathrm{E}+01$ & $0.0 \mathrm{E}+00$ & $9.6 \mathrm{E}+01$ & $2.2 \mathrm{E}+01$ & $7.8 \mathrm{E}+03$ & $3.0 \mathrm{E}+02$ & & $3.0 \mathrm{E}+01$ & $1.1 \mathrm{E}+02$ & $7.4 \mathrm{E}+03$ \\
\hline $15 \mathrm{H}$ & & 0 & $8.6 \mathrm{E}+04$ & 2 & $1.8 \mathrm{E}+02$ & $2 \mathrm{E}+03$ & $7.7 \mathrm{E}+02$ & $\mathrm{DE}$ & +00 & $3.1 \mathrm{E}+02$ & $0.0 \mathrm{E}+00$ & $4.7 \mathrm{E}+02$ & $1.0 \mathrm{E}+02$ & $2.8 \mathrm{E}+04$ & $6.3 \mathrm{E}+03$ & 4.7 & $1.5 \mathrm{E}+02$ & $6.3 \mathrm{E}+02$ & $6.6 \mathrm{E}+03$ \\
\hline $16 \mathrm{H}$ & & & & & & & & & & & & & & & & & & & \\
\hline $21 \mathrm{H}$ & & & $1.5 \mathrm{E}+03$ & & $5.2 \mathrm{E}-02$ & 00 & & & & $6.7 \mathrm{E}+02$ & $0.0 \mathrm{E}+0$ & $8.7 \mathrm{E}+01$ & $2.3 \mathrm{E}+01$ & $2.6 \mathrm{E}+04$ & $1.2 \mathrm{E}+03$ & & $2.0 \mathrm{E}+02$ & $3.5 \mathrm{E}+02$ & $2.4 \mathrm{E}+00$ \\
\hline 221 & & 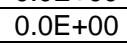 & $1.9 \mathrm{E}+03$ & 2 & $0.0 \mathrm{E}+00$ & $0 \mathrm{E}+00$ & & & $0.0 \mathrm{E}+00$ & $1.0 \mathrm{E}+03$ & $0.0 \mathrm{E}+00$ & $1.3 \mathrm{E}+02$ & $3.4 \mathrm{E}+01$ & $3.9 \mathrm{E}+04$ & $1.8 \mathrm{E}+03$ & & $3.1 \mathrm{E}+02$ & $5.4 \mathrm{E}+02$ & $0.0 \mathrm{E}+00$ \\
\hline 30 & & & & & $6.0 \mathrm{E}-01$ & & & & & & & & & & & & & $1.8 \mathrm{E}+00$ & $3.0 \mathrm{E}+01$ \\
\hline $32 \mathrm{H}$ & & & & & $3.7 \mathrm{E}+02$ & & & & & & & & & & & & & & \\
\hline $35 \mathrm{~h}$ & & & 4 & & $\mathrm{E}+02$ & $J_{0}$ & & & & & & .4 & 1.6 & & & & $1.4 \mathrm{E}+02$ & $8.8 \mathrm{E}+02$ & $4.6 \mathrm{E}+03$ \\
\hline & & & & & & & & & & & & & & & & & & $5.5 \mathrm{E}-01$ & \\
\hline $39 \mathrm{H}$ & & 0 & & & 2. & 3 & 5.1 & & & 3 & & 7 & $1.6 \mathrm{E}+02$ & 7.6 & $9.5 \mathrm{E}+03$ & & $5.1 \mathrm{E}+02$ & $1.3 \mathrm{E}+03$ & $1.9 \mathrm{E}+04$ \\
\hline 411 & & & & & & & & & & & & & & & & & & & \\
\hline 43 & int & & & & $3.1 \mathrm{E}$ & $8.8 \mathrm{E}+01$ & & & & 1.0 & & 1.45 & $3.6 \mathrm{E}+01$ & & 1.91 & & 3.0 & $5.4 \mathrm{E}+02$ & $1.1 \mathrm{E}+02$ \\
\hline Total & & $1.5 \mathrm{E}+02$ & $5.3 \mathrm{E}+05$ & $4.4 \mathrm{E}+03$ & $1.7 \mathrm{E}+03$ & $3.7 \mathrm{E}+04$ & $3.9 \mathrm{E}+04$ & $1.3 \mathrm{E}+02$ & $2.4 \mathrm{E}+02$ & $1.1 \mathrm{E}+04$ & $9.2 \mathrm{E}+00$ & $5.5 \mathrm{E}+03$ & $1.3 \mathrm{E}+03$ & $5.7 \mathrm{E}+05$ & $6.8 \mathrm{E}+04$ & $5.6 \mathrm{E}+03$ & $3.7 \mathrm{E}+03$ & $9.8 \mathrm{E}+03$ & $9.3 \mathrm{E}+04$ \\
\hline$\%$ of $\$$ & & $8.9 \mathrm{E}-03$ & $3.2 \mathrm{E}+01$ & 2.6E-01 & $1.0 \mathrm{E}-01$ & $2.2 \mathrm{E}+00$ & $2.3 \mathrm{E}+00$ & $7.9 \mathrm{E}-03$ & $1.4 \mathrm{E}-02$ & $6.3 \mathrm{E}-01$ & $5.5 \mathrm{E}-04$ & 3.3E-01 & $7.7 \mathrm{E}-02$ & $3.4 \mathrm{E}+01$ & $4.1 \mathrm{E}+00$ & $3.3 \mathrm{E}-01$ & $2.2 \mathrm{E}-01$ & $5.8 \mathrm{E}-01$ & $5.6 \mathrm{E}+00$ \\
\hline
\end{tabular}

\begin{tabular}{|c|c|c|c|c|c|c|c|c|c|c|c|c|c|c|c|c|c|c|c|}
\hline Tank \# & 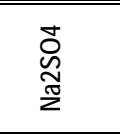 & $\begin{array}{l}\text { Dे } \\
\text { o. } \\
\text { In }\end{array}$ & $\begin{array}{l}\overline{\tilde{z}} \\
\tilde{z}\end{array}$ & 峁 & ָू & 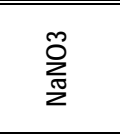 & $\begin{array}{l}\frac{T}{0} \\
\frac{\pi}{2}\end{array}$ & 㐱 & 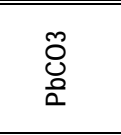 & 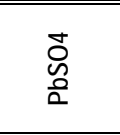 & 을 & $\stackrel{\widetilde{O}}{\widetilde{\alpha}}$ & ণั & 芯 & ర్ & 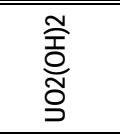 & $\begin{array}{l}\frac{\widetilde{\widetilde{J}}}{\mathrm{O}} \\
\frac{\mathrm{N}}{\mathrm{N}}\end{array}$ & 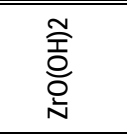 & $\begin{array}{c}\text { Total } \\
\text { SS } \\
\text { Mass, } \\
\text { kg }\end{array}$ \\
\hline$\overline{9 \mathrm{H}}$ & $\mathrm{DE}+00$ & $\overline{\mathrm{E}+00}$ & $\mathrm{E}+02$ & $E+00$ & +00 & $7 \overline{E+02}$ & $6 \mathrm{E}+02$ & $1.0 \mathrm{E}+03$ & $\mathrm{DE}+01$ & $3 \overline{E+01}$ & $1 \mathrm{E}+01$ & $8 \mathrm{E}+01$ & $1.8 \mathrm{E}+02$ & $2.0 \mathrm{E}+01$ & $0.0 \mathrm{E}+00$ & $1 \mathrm{E}+03$ & $4.6 \mathrm{E}+01$ & $8.5 \mathrm{E}+01$ & $1.5 \mathrm{E}+04$ \\
\hline $10 \mathrm{H}$ & $E+00$ & $E+00$ & $E+02$ & $\mathrm{E}+00$ & +00 & $\mathrm{E}+02$ & $7 \mathrm{E}+02$ & $2.2 \mathrm{E}+03$ & $\mathrm{E}+01$ & $\mathrm{FE}+01$ & $6 \mathrm{E}+01$ & $2 \mathrm{E}+02$ & $3.2 \mathrm{E}+02$ & $3.4 \mathrm{E}+01$ & $0.0 \mathrm{E}+00$ & $2.2 \mathrm{E}+03$ & $7.9 \mathrm{E}+01$ & $1.5 \mathrm{E}+02$ & $3.1 \mathrm{E}+04$ \\
\hline $11 \mathrm{H}$ & $\mathrm{E}+02$ & $1 \mathrm{E}+01$ & $7 \mathrm{E}+02$ & $7 \mathrm{E}+02$ & $0 \mathrm{E}+00$ & $9 \mathrm{E}+03$ & $5 \mathrm{E}+03$ & $3 \mathrm{E}+02$ & $.6 \mathrm{E}+01$ & $5 \mathrm{E}+02$ & $2.0 \mathrm{E}+02$ & $7.6 \mathrm{E}+01$ & $1.1 \mathrm{E}+04$ & $1.7 \mathrm{E}+02$ & $6.2 \mathrm{E}+02$ & $9.5 \mathrm{E}+02$ & $1.2 \mathrm{E}+02$ & $3.2 \mathrm{E}+02$ & $2.1 \mathrm{E}+05$ \\
\hline $12 \mathrm{r}$ & & 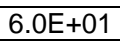 & 50 & & & & & & & & & & & & & $6 \mathrm{E}+03$ & & $8 \mathrm{E}+02$ & \\
\hline $13 \mathrm{H}$ & $E+01$ & $8.3 \mathrm{E}+00$ & $5.2 \mathrm{E}+03$ & $7 \mathrm{E}+01$ & $E+00$ & $E+03$ & $1.2 \mathrm{E}+04$ & & $2.3 \mathrm{E}+02$ & $2.0 \mathrm{E}$ & $8.7 \mathrm{E}+02$ & $8.9 \mathrm{E}+00$ & 1.3E- & $1.8 \mathrm{E}$ & & $1.5 \mathrm{E}+03$ & $1.4 \mathrm{E}+01$ & $2.0 \mathrm{E}+03$ & +05 \\
\hline $14 \mathrm{H}$ & $E+01$ & $9 \mathrm{E}+00$ & $7.8 \mathrm{E}+01$ & $5 \mathrm{E}+01$ & $\mathrm{DE}+00$ & $\mathrm{E}+02$ & $7.7 \mathrm{E}+02$ & 03 & $5.7 \mathrm{E}+01$ & $3.4 \mathrm{E}+01$ & $2.5 \mathrm{E}+01$ & $4.4 \mathrm{E}+01$ & $4.0 \mathrm{E}+02$ & $3.6 \mathrm{E}+01$ & $7.3 \mathrm{E}+02$ & $2.0 \mathrm{E}+03$ & $2.9 \mathrm{E}+01$ & $1.0 \mathrm{E}+02$ & $2.7 \mathrm{E}+04$ \\
\hline $15 \mathrm{H}$ & $\mathrm{E}+02$ & $5.8 \mathrm{E}+01$ & $2.4 \mathrm{E}+02$ & $4.6 \mathrm{E}+02$ & $\mathrm{DE}+00$ & $4 \mathrm{E}+03$ & $3.2 \mathrm{E}+03$ & $3.9 \mathrm{E}+01$ & $1.1 \mathrm{E}+01$ & $1.2 \mathrm{E}+02$ & $1.3 \mathrm{E}+02$ & $6.2 \mathrm{E}+01$ & $9.2 \mathrm{E}+03$ & $1.3 \mathrm{E}+02$ & $1.7 \mathrm{E}+04$ & $5.5 \mathrm{E}+01$ & $9.5 \mathrm{E}+01$ & $5.9 \mathrm{E}+02$ & $1.7 \mathrm{E}+05$ \\
\hline $16 \mathrm{H}$ & & & & & & & & & & & & & 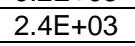 & & & $.0 \mathrm{E}$ & & $2.4 \mathrm{E}+02$ & $7.1 \mathrm{E}+04$ \\
\hline $21 \mathrm{H}$ & $\mathrm{E}-01$ & $1.7 \mathrm{E}-02$ & $8.1 \mathrm{E}+02$ & $1.4 \mathrm{E}-01$ & $\mathrm{E}+$ & $E+02$ & $3 \mathrm{E}+03$ & & $3.6 \mathrm{E}$ & $3.2 \mathrm{E}$ & $1.3 \mathrm{E}+02$ & $1.8 \mathrm{E}$ & $2.7 \mathrm{E}$ & $2.5 \mathrm{E}$ & & $1.8 \mathrm{E}+01$ & $2.8 \mathrm{E}-02$ & $3.0 \mathrm{E}+02$ & $3.6 \mathrm{E}+04$ \\
\hline $22 \mathrm{H}$ & $E+00$ & $0.0 \mathrm{E}+00$ & $1.2 \mathrm{E}+03$ & $0.0 \mathrm{E}+00$ & & 02 & 03 & & & & $2.0 \mathrm{E}+02$ & $0.0 \mathrm{E}$ & $0.0 \mathrm{E}$ & & & $1.6 \mathrm{E}+02$ & $0.0 \mathrm{E}+00$ & $4.6 \mathrm{E}+02$ & $5.5 \mathrm{E}+04$ \\
\hline $30 \mathrm{H}$ & $E+00$ & $2.0 \mathrm{E}-01$ & $0.0 \mathrm{E}+00$ & & & & 9. & & & & $2.9 \mathrm{E}-01$ & $2.1 \mathrm{E}$ & & & & $3.1 \mathrm{E}-01$ & $3.3 \mathrm{E}-01$ & $1.7 \mathrm{E}+00$ & +02 \\
\hline $32 \mathrm{H}$ & $3+$ & $1.2 \mathrm{E}$ & & & & & & & & & & & & & & & & $115+0$ & \\
\hline $35 \mathrm{H}$ & 3 & 9. & & & & & & & & & & & & & & 1.5 & & +02 & \\
\hline 36 & & & $0.0 \mathrm{E}-$ & & & & & & $0.0 \mathrm{E}$ & & & & & & & $3.1 \mathrm{E}-01$ & 9.9E-02 & $5.2 \mathrm{E}-01$ & $1.6 \mathrm{E}+02$ \\
\hline $39 \mathrm{H}$ & 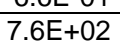 & 6.91 & $1.6 \mathrm{E}$ & 2 & & 3 & $6.9 \mathrm{E}$ & $0.0 \mathrm{E}$ & $7.3 \mathrm{E}$ & & 3.7 & & & 2.0 & & $4.3 \mathrm{E}+02$ & 1.1 & $1.2 \mathrm{E}+03$ & $1.8 \mathrm{E}+05$ \\
\hline $41 \mathrm{H}$ & & & & & & & & & & & & & & & & & & & \\
\hline $43 \mathrm{~K}$ & 1 & & $1.2 \mathrm{E}+03$ & & & & $2.8 \mathrm{E}-$ & & 5.5E & & $2.0 \mathrm{E}$ & & & $3.9 \mathrm{E}$ & & $5.6 \mathrm{E}$ & 2.0E & $4.7 \mathrm{E}+02$ & \\
\hline$\pi$ & $5.5 \mathrm{E}+03$ & $5.1 \mathrm{E}+02$ & $1.2 \mathrm{E}+04$ & $4.0 \mathrm{E}+03$ & $4 \mathrm{E}+01$ & $5.9 \mathrm{E}+04$ & $5.2 \mathrm{E}+04$ & $1.3 \mathrm{E}+04$ & $6.6 \mathrm{E}+02$ & $9 \mathrm{E}+03$ & $2.7 \mathrm{E}+03$ & $9.0 \mathrm{E}+02$ & 8.1 & $1.6 \mathrm{E}+03$ & & $1.1 \mathrm{E}+04$ & $1.1 \mathrm{E}+03$ & $9.1 \mathrm{E}+03$ & $1.7 \mathrm{E}+06$ \\
\hline & $3.3 \mathrm{E}-01$ & $230 \mathrm{~F} 02$ & 70250 & 21501 & 0 & 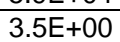 & 0.25 & $70 \div$ & $20=0$ & 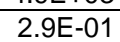 & 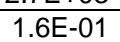 & & $5+00$ & & & $6.8 \mathrm{E}-01$ & $6.4 \mathrm{E}-02$ & . & \\
\hline
\end{tabular}


Table 3-3. Estimated Curies of Radionuclides in Sludge Solids (SS) Received into the F Area Waste Tanks (Curies have been adjusted for decay)

\begin{tabular}{|c|c|c|c|c|c|c|c|c|c|c|c|c|c|c|c|c|c|c|c|}
\hline Tank \# & 今ٌ & $\begin{array}{l}\text { ò } \\
\dot{d}\end{array}$ & 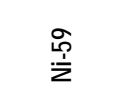 & 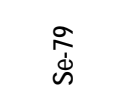 & $\begin{array}{l}\text { क्षे } \\
\dot{\vdots 亠}\end{array}$ & 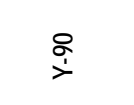 & 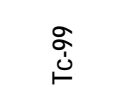 & 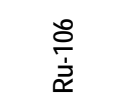 & 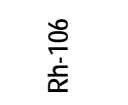 & $\begin{array}{l}\stackrel{\text { I }}{1} \\
\text { के }\end{array}$ & $\begin{array}{l}\stackrel{\mathscr{I}}{\leftrightarrows} \\
\dot{\omega}\end{array}$ & $\underset{乛}{\text { İ }}$ & 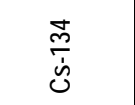 & $\begin{array}{l}\stackrel{\mathscr{m}}{\dot{S}} \\
\text { Un }\end{array}$ & 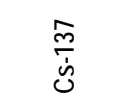 & 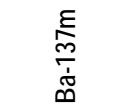 & $\begin{array}{l}\vec{J} \\
\dot{d} \\
\dot{U}\end{array}$ & 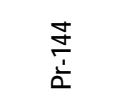 & 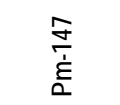 \\
\hline $1 \mathrm{~F}$ & $5.2 \mathrm{E}-02$ & $3.3 \mathrm{E}$ & 1 & $5 E+01$ & 36 & $1.9 \mathrm{E}$ & 3 & $1.8 \mathrm{E}-07$ & $1.8 \mathrm{E}-07$ & $8.6 \mathrm{E}$ & $1.2 \mathrm{E}$ & $\overline{c 5.4 \mathrm{E}-03}$ & $4.5 \mathrm{E}-03$ & $7.6 \mathrm{E}-01$ & $1.4 \mathrm{E}$ & $1.3 \mathrm{E}+05$ & $4 \mathrm{E}-11$ & 4.4E-11 & $1.1 \mathrm{E}+02$ \\
\hline $2 \mathrm{~F}$ & $2.9 \mathrm{E}-02$ & $5.5 \mathrm{E}+01$ & $3.4 \mathrm{E}$ & $2.3 \mathrm{E}+01$ & 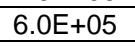 & & $4.0 \mathrm{E}+02$ & & & & & & & & & $4.1 \mathrm{E}+04$ & & & \\
\hline $3 \mathrm{~F}$ & $3.0 \mathrm{E}-02$ & $7.5 \mathrm{E}+01$ & $3.5 \mathrm{E}+01$ & $2.4 \mathrm{E}+01$ & $6.6 \mathrm{E}+05$ & $6.6 \mathrm{E}+05$ & $4.2 \mathrm{E}+02$ & $2.3 \mathrm{E}-09$ & $2.3 \mathrm{E}-09$ & $1.1 \mathrm{E}+00$ & $4.5 \mathrm{E}+01$ & $2.0 \mathrm{E}-03$ & $3.7 \mathrm{E}-04$ & $2.8 \mathrm{E}-01$ & $4.7 \mathrm{E}+04$ & $4.5 \mathrm{E}+04$ & $1.9 \mathrm{E}-13$ & $1.9 \mathrm{E}-13$ & $1.3 \mathrm{E}+01$ \\
\hline $4 \mathrm{~F}$ & $3.3 \mathrm{E}-03$ & $1.7 \mathrm{E}+03$ & $1.0 \mathrm{E}+02$ & $7.2 \mathrm{E}+01$ & $2.7 \mathrm{E}+06$ & $2.7 \mathrm{E}+06$ & $1.2 \mathrm{E}+03$ & $1.9 \mathrm{E}-03$ & $1.9 \mathrm{E}-03$ & $2.4 \mathrm{E}+02$ & $1.3 \mathrm{E}+02$ & $5.9 \mathrm{E}-03$ & $4.1 \mathrm{E}-01$ & $8.3 \mathrm{E}-01$ & $1.9 \mathrm{E}+05$ & $1.8 \mathrm{E}+05$ & $8.1 \mathrm{E}-06$ & $8.1 \mathrm{E}-06$ & $3.6 \mathrm{E}+03$ \\
\hline $5 \mathrm{~F}$ & $4.4 \mathrm{E}-02$ & $5.4 \mathrm{E}+02$ & $9.8 \mathrm{E}+01$ & $6.9 \mathrm{E}+01$ & $2.2 \mathrm{E}+06$ & $2.2 \mathrm{E}+06$ & $1.2 \mathrm{E}+03$ & $1.9 \mathrm{E}-06$ & $1.9 \mathrm{E}-06$ & $2.2 \mathrm{E}+01$ & $1.3 \mathrm{E}+02$ & $5.7 \mathrm{E}-03$ & $1.5 \mathrm{E}-02$ & $7.9 \mathrm{E}-01$ & $1.6 \mathrm{E}+05$ & $1.5 \mathrm{E}+05$ & $8.9 \mathrm{E}-10$ & $8.9 \mathrm{E}-10$ & $2.9 \mathrm{E}+02$ \\
\hline $6 \mathrm{~F}$ & $0.0 \mathrm{E}+00$ & $8.1 \mathrm{E}+02$ & $9.3 \mathrm{E}+01$ & $6.7 \mathrm{E}+01$ & $2.3 \mathrm{E}+06$ & $2.3 \mathrm{E}+06$ & $1.2 \mathrm{E}+03$ & $1.2 \mathrm{E}-05$ & $1.2 \mathrm{E}-05$ & $4.4 \mathrm{E}+01$ & $1.2 \mathrm{E}+02$ & $5.5 \mathrm{E}-03$ & $3.8 \mathrm{E}-02$ & $7.7 \mathrm{E}-01$ & $1.6 \mathrm{E}+05$ & $1.6 \mathrm{E}+05$ & & & $6.0 \mathrm{E}+02$ \\
\hline $7 \mathrm{~F}$ & $6.3 \mathrm{E}-01$ & $3.8 \mathrm{E}+02$ & $1.7 \mathrm{E}+02$ & $9.6 \mathrm{E}+01$ & $2.7 \mathrm{E}+06$ & $2.7 \mathrm{E}+06$ & $1.7 \mathrm{E}+03$ & $1.4 \mathrm{E}-07$ & $1.4 \mathrm{E}-07$ & $8.3 \mathrm{E}+00$ & $1.8 \mathrm{E}+02$ & $7.9 \mathrm{E}-03$ & $4.0 \mathrm{E}-03$ & $1.1 \mathrm{E}+00$ & $1.9 \mathrm{E}+05$ & $1.8 \mathrm{E}+05$ & $3.6 \mathrm{E}-11$ & $3.6 \mathrm{E}-11$ & $1.1 \mathrm{E}+02$ \\
\hline $8 \mathrm{~F}$ & $2.0 \mathrm{E}-01$ & $1.1 \mathrm{E}+03$ & $1.0 \mathrm{E}+02$ & $6.7 \mathrm{E}+01$ & $2.4 \mathrm{E}+06$ & $2.4 \mathrm{E}+06$ & $1.2 \mathrm{E}+03$ & $2.0 \mathrm{E}-04$ & $2.0 \mathrm{E}-04$ & $9.7 \mathrm{E}+01$ & $1.2 \mathrm{E}+02$ & $5.5 \mathrm{E}-03$ & $1.2 \mathrm{E}-01$ & $7.8 \mathrm{E}-01$ & $1.7 \mathrm{E}+05$ & $1.6 \mathrm{E}+05$ & $7.9 \mathrm{E}-07$ & $7.9 \mathrm{E}-07$ & $1.4 \mathrm{E}+03$ \\
\hline $17 \mathrm{~F}$ & $5.7 \mathrm{E}-01$ & $5.9 \mathrm{E}+01$ & $3.4 \mathrm{E}+01$ & $2.8 \mathrm{E}+00$ & $1.1 \mathrm{E}+05$ & $1.1 \mathrm{E}+05$ & $4.8 \mathrm{E}+01$ & $1.0 \mathrm{E}-05$ & $1.0 \mathrm{E}-05$ & $5.6 \mathrm{E}+00$ & $5.2 \mathrm{E}+00$ & $2.3 \mathrm{E}-04$ & $7.0 \mathrm{E}-03$ & $3.2 \mathrm{E}-02$ & $7.5 \mathrm{E}+03$ & $7.1 \mathrm{E}+03$ & $2.5 \mathrm{E}-08$ & $2.5 \mathrm{E}-08$ & $8.1 \mathrm{E}+01$ \\
\hline $18 \mathrm{~F}$ & $6.2 \mathrm{E}-01$ & $6.4 \mathrm{E}+01$ & $3.7 \mathrm{E}+01$ & $3.0 \mathrm{E}+00$ & $1.2 \mathrm{E}+05$ & $1.2 \mathrm{E}+05$ & $5.3 \mathrm{E}+01$ & $1.2 \mathrm{E}-05$ & $1.2 \mathrm{E}-05$ & $6.1 \mathrm{E}+00$ & $5.6 \mathrm{E}+00$ & $2.5 \mathrm{E}-04$ & $7.7 \mathrm{E}-03$ & $3.5 \mathrm{E}-02$ & $8.2 \mathrm{E}+03$ & $7.7 \mathrm{E}+03$ & $2.7 \mathrm{E}-08$ & $2.7 \mathrm{E}-08$ & $8.8 \mathrm{E}+01$ \\
\hline $19 \mathrm{~F}$ & $2.1 \mathrm{E}-03$ & $2.7 \mathrm{E}-01$ & $1.2 \mathrm{E}-01$ & $1.0 \mathrm{E}-02$ & $4.1 \mathrm{E}$ & $4.1 \mathrm{E}+02$ & $1.7 \mathrm{E}-01$ & $3.1 \mathrm{E}-08$ & $3.1 \mathrm{E}-08$ & $2.6 \mathrm{E}-02$ & $1.9 \mathrm{E}-02$ & & $3.2 \mathrm{E}-05$ & $1.2 \mathrm{E}-04$ & $2.9 \mathrm{E}+01$ & $2.7 \mathrm{E}+01$ & $4.8 \mathrm{E}-11$ & $4.8 \mathrm{E}-11$ & $3.8 \mathrm{E}-01$ \\
\hline $26 \mathrm{~F}$ & $5.5 \mathrm{E}-01$ & $3.8 \mathrm{E}+$ & $3.2 \mathrm{E}+01$ & $2.7 \mathrm{E}-$ & $1.5 \mathrm{E}+05$ & $1.5 \mathrm{E}+05$ & $4.6 \mathrm{E}+01$ & $6.0 \mathrm{E}-01$ & $6.0 \mathrm{E}-01$ & $2.1 \mathrm{E}+02$ & $4.9 \mathrm{E}$ & $2.2 \mathrm{E}-04$ & $9.7 \mathrm{E}-01$ & $3.1 \mathrm{E}-02$ & $1.0 \mathrm{E}+04$ & $9.5 \mathrm{E}+03$ & $6.7 \mathrm{E}-02$ & $6.7 \mathrm{E}-02$ & $3.6 \mathrm{E}+03$ \\
\hline $33 \mathrm{~F}$ & $1.2 \mathrm{E}-01$ & $2.3 \mathrm{E}+04$ & $1.8 \mathrm{E}+02$ & $1.2 \mathrm{E}+02$ & $7.0 \mathrm{E}+06$ & $7.0 \mathrm{E}+06$ & $2.1 \mathrm{E}+03$ & $5.9 \mathrm{E}+01$ & $5.9 \mathrm{E}+01$ & $1.8 \mathrm{E}+04$ & $2.3 \mathrm{E}+02$ & $1.0 \mathrm{E}-02$ & $9.5 \mathrm{E}+01$ & $1.4 \mathrm{E}+00$ & $4.8 \mathrm{E}+05$ & $4.6 \mathrm{E}+05$ & $5.2 \mathrm{E}+00$ & $5.2 \mathrm{E}+00$ & $3.1 \mathrm{E}+05$ \\
\hline $34 \mathrm{~F}$ & $0.0 \mathrm{E}+00$ & $1.3 \mathrm{E}+04$ & $1.7 \mathrm{E}+02$ & $1.2 \mathrm{E}+02$ & $6.2 \mathrm{E}+06$ & $6.2 \mathrm{E}+06$ & $2.1 \mathrm{E}+03$ & $2.0 \mathrm{E}+00$ & $2.0 \mathrm{E}+00$ & $5.6 \mathrm{E}+03$ & $2.2 \mathrm{E}+02$ & $9.8 \mathrm{E}-03$ & $1.9 \mathrm{E}+01$ & $1.4 \mathrm{E}+00$ & $4.3 \mathrm{E}+05$ & $4.1 \mathrm{E}+05$ & $6.3 \mathrm{E}-02$ & $6.3 \mathrm{E}-02$ & $9.1 \mathrm{E}+04$ \\
\hline $47 \mathrm{~F}$ & $4.3 \mathrm{E}-01$ & $2.2 \mathrm{E}+02$ & $2.5 \mathrm{E}+01$ & $2.1 \mathrm{E}+00$ & $1.1 \mathrm{E}+05$ & $1.1 \mathrm{E}+05$ & $3.6 \mathrm{E}+01$ & $1.1 \mathrm{E}-02$ & $1.1 \mathrm{E}-02$ & $8.0 \mathrm{E}+01$ & $3.9 \mathrm{E}+00$ & $1.7 \mathrm{E}-04$ & $2.4 \mathrm{E}-01$ & $2.4 \mathrm{E}-02$ & $7.6 \mathrm{E}+03$ & $7.2 \mathrm{E}+03$ & $1.8 \mathrm{E}-04$ & $1.8 \mathrm{E}-04$ & $1.3 \mathrm{E}+03$ \\
\hline Total Ci & $3.3 \mathrm{E}+00$ & $4.2 \mathrm{E}+04$ & $1.2 \mathrm{E}+03$ & $7.3 \mathrm{E}+02$ & $2.9 \mathrm{E}+07$ & $2.9 \mathrm{E}+07$ & $1.3 \mathrm{E}+04$ & $6.2 \mathrm{E}+01$ & $6.2 \mathrm{E}+01$ & $2.4 \mathrm{E}+04$ & $1.4 \mathrm{E}+03$ & $6.0 \mathrm{E}-02$ & $1.2 \mathrm{E}+02$ & $8.5 \mathrm{E}+00$ & $2.0 \mathrm{E}+06$ & $1.9 \mathrm{E}+06$ & $5.3 \mathrm{E}+00$ & $5.3 \mathrm{E}+00$ & $4.1 \mathrm{E}+05$ \\
\hline $\mathrm{Ci} / \mathrm{kg} \mathrm{SS}$ & $1.4 \mathrm{E}-06$ & $1.8 \mathrm{E}-02$ & $5.0 \mathrm{E}-04$ & $3.0 \mathrm{E}-04$ & $1.2 \mathrm{E}+01$ & $1.2 \mathrm{E}+01$ & $5.3 \mathrm{E}-03$ & $2.6 \mathrm{E}-05$ & $2.6 \mathrm{E}-05$ & $1.0 \mathrm{E}-02$ & $5.7 \mathrm{E}-04$ & $2.5 \mathrm{E}-08$ & $4.8 \mathrm{E}-05$ & $3.5 \mathrm{E}-06$ & $8.5 \mathrm{E}-01$ & $8.0 \mathrm{E}-01$ & $2.2 \mathrm{E}-06$ & $2.2 \mathrm{E}-06$ & $1.7 \mathrm{E}-01$ \\
\hline
\end{tabular}

\begin{tabular}{|c|c|c|c|c|c|c|c|c|c|c|c|c|c|c|c|c|c|c|c|}
\hline Tank \# & $\begin{array}{l}\text { 荣 } \\
\text { 离 }\end{array}$ & 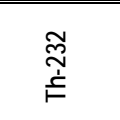 & $\underset{\widetilde{N}}{3}$ & ֶָָ ָָ & 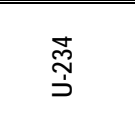 & $\stackrel{\mathscr{N}}{\circlearrowleft}$ & 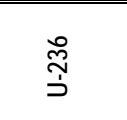 & 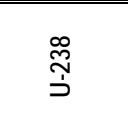 & $\begin{array}{l}\hat{\tilde{\aleph}} \\
\hat{\dot{\omega}}\end{array}$ & 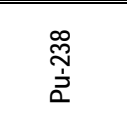 & 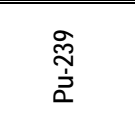 & $\begin{array}{l}\stackrel{9}{\dddot{a}} \\
\grave{a}\end{array}$ & 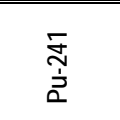 & 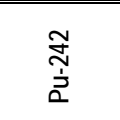 & 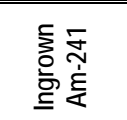 & 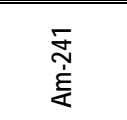 & 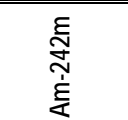 & 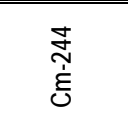 & 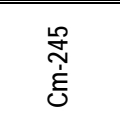 \\
\hline$\overline{1 F}$ & $2 \mathrm{E}+03$ & +00 & $6 \overline{6 E-02}$ & $\overline{\mathrm{DE}+00}$ & $\overline{2}$ & $7.7 \mathrm{E}-0$ & 0 & $1.9 \mathrm{E}+00$ & $\overline{8 \mathrm{E}+00}$ & $2.2 \mathrm{E}+03$ & $5.8 \mathrm{E}+0$ & $1.3 \mathrm{E}+02$ & $\overline{1 E+02}$ & $2.7 \mathrm{E}-02$ & $9 \mathrm{E}+02$ & $3 \mathrm{E}+03$ & $.0 \mathrm{E}+01$ & $1.2 \mathrm{E}+00$ & $\begin{array}{l}1.9 \mathrm{E}-06 \\
\end{array}$ \\
\hline $2 \mathrm{~F}$ & $E+02$ & $E+00$ & $\mathrm{E}-02$ & $\mathrm{E}+00$ & $E+00$ & $8.1 \mathrm{E}-03$ & $\mathrm{E}+00$ & $0 \mathrm{E}-01$ & $1 E+00$ & $1.4 \mathrm{E}+03$ & $2.0 \mathrm{E}+02$ & $4.4 \mathrm{E}+01$ & $3 \mathrm{E}+02$ & $9.1 \mathrm{E}-03$ & $3 \mathrm{E}+01$ & $2.9 \mathrm{E}+03$ & $3.5 \mathrm{E}+00$ & $3.4 \mathrm{E}-01$ & $6.6 \mathrm{E}-07$ \\
\hline $3 \mathrm{~F}$ & $6.3 \mathrm{E}+02$ & $0.0 \mathrm{E}+00$ & $1.7 \mathrm{E}-02$ & $0 \mathrm{E}+00$ & $0.0 \mathrm{E}+00$ & $2.5 \mathrm{E}-02$ & $0.0 \mathrm{E}+00$ & $6.3 \mathrm{E}-01$ & $4.7 \mathrm{E}+00$ & $2.1 \mathrm{E}+03$ & $3.0 \mathrm{E}+02$ & $6.6 \mathrm{E}+01$ & $2.4 \mathrm{E}+02$ & $1.4 \mathrm{E}-02$ & $9.6 \mathrm{E}+01$ & $3.1 \mathrm{E}+03$ & $3.7 \mathrm{E}+00$ & $3.9 \mathrm{E}-01$ & $6.9 \mathrm{E}-07$ \\
\hline $4 \mathrm{~F}$ & $5.7 \mathrm{E}+03$ & $0.0 \mathrm{E}+00$ & $5.5 \mathrm{E}-02$ & $0 \mathrm{E}+00$ & $0.0 \mathrm{E}+00$ & $7.8 \mathrm{E}-02$ & $0.0 \mathrm{E}+00$ & $3.3 \mathrm{E}+00$ & $3.0 \mathrm{E}+00$ & $6.1 \mathrm{E}+02$ & $6.0 \mathrm{E}+02$ & $1.3 \mathrm{E}+02$ & $8.1 \mathrm{E}+02$ & $2.7 \mathrm{E}-02$ & $1.9 \mathrm{E}+02$ & $9.7 \mathrm{E}+03$ & $1.2 \mathrm{E}+01$ & $1.6 \mathrm{E}+04$ & $6.9 \mathrm{E}-01$ \\
\hline $5 \mathrm{~F}$ & $3.0 \mathrm{E}+03$ & $0.0 \mathrm{E}+00$ & $5.0 \mathrm{E}-02$ & $0.0 \mathrm{E}+00$ & $0.0 \mathrm{E}+00$ & $1.0 \mathrm{E}-01$ & $0.0 \mathrm{E}+00$ & $2.5 \mathrm{E}+00$ & $4.2 \mathrm{E}+00$ & $2.9 \mathrm{E}+03$ & $4.8 \mathrm{E}+02$ & $1.2 \mathrm{E}+02$ & $5.5 \mathrm{E}+02$ & $3.4 \mathrm{E}-02$ & $1.8 \mathrm{E}+02$ & $8.8 \mathrm{E}+03$ & $1.1 \mathrm{E}+01$ & $1.4 \mathrm{E}+00$ & $1.9 \mathrm{E}-06$ \\
\hline $6 \mathrm{~F}$ & $3.9 \mathrm{E}+03$ & $0.0 \mathrm{E}+00$ & $5.0 \mathrm{E}-02$ & $0 \mathrm{E}+00$ & $0.0 \mathrm{E}+00$ & $6.6 \mathrm{E}-02$ & $0.0 \mathrm{E}+00$ & $2.5 \mathrm{E}+00$ & $8.6 \mathrm{E}-01$ & $0.0 \mathrm{E}+00$ & $2.5 \mathrm{E}+02$ & $8.8 \mathrm{E}+01$ & $7.1 \mathrm{E}+02$ & $1.7 \mathrm{E}-01$ & $1.9 \mathrm{E}+02$ & $8.7 \mathrm{E}+03$ & $1.1 \mathrm{E}+01$ & $1.4 \mathrm{E}+03$ & $1.9 \mathrm{E}-06$ \\
\hline $7 \mathrm{~F}$ & $2.8 \mathrm{E}+03$ & $0.0 \mathrm{E}+00$ & $7.2 \mathrm{E}-02$ & $0 \mathrm{E}+00$ & $0.0 \mathrm{E}+00$ & $3.8 \mathrm{E}-01$ & $0.0 \mathrm{E}+00$ & $9.2 \mathrm{E}+00$ & $8.4 \mathrm{E}+00$ & $2.1 \mathrm{E}+04$ & $3.7 \mathrm{E}+03$ & $8.8 \mathrm{E}+02$ & $7.2 \mathrm{E}+02$ & $2.8 \mathrm{E}-01$ & $2.9 \mathrm{E}+02$ & $1.2 \mathrm{E}+04$ & $1.4 \mathrm{E}+01$ & $1.6 \mathrm{E}+00$ & $2.7 \mathrm{E}-06$ \\
\hline $8 \mathrm{~F}$ & $4.6 \mathrm{E}+03$ & $0.0 \mathrm{E}+00$ & $5.3 \mathrm{E}-02$ & $\mathrm{DE}+00$ & $0.0 \mathrm{E}+00$ & $1.5 \mathrm{E}-01$ & & $6.9 \mathrm{E}+00$ & $3.1 \mathrm{E}+00$ & & & $4.7 \mathrm{E}$ & & & $5.4 \mathrm{E}+02$ & $9.7 \mathrm{E}+03$ & $1.1 \mathrm{E}+01$ & $1.9 \mathrm{E}+04$ & $3.4 \mathrm{E}-01$ \\
\hline $17 \mathrm{~F}$ & $2.2 \mathrm{E}+02$ & $0.0 \mathrm{E}+00$ & $7.5 \mathrm{E}-03$ & $0 \mathrm{E}+00$ & $0.0 \mathrm{E}+00$ & $6.8 \mathrm{E}-02$ & $0.0 \mathrm{E}+00$ & $4.2 \mathrm{E}+00$ & $0.0 \mathrm{E}+00$ & $1.6 \mathrm{E}+04$ & $2.5 \mathrm{E}+03$ & $6.5 \mathrm{E}+02$ & $5.8 \mathrm{E}+03$ & $9.4 \mathrm{E}-01$ & $1.1 \mathrm{E}+03$ & $3.4 \mathrm{E}+01$ & $0.0 \mathrm{E}+00$ & $1.2 \mathrm{E}+04$ & $8.0 \mathrm{E}-08$ \\
\hline $18 \mathrm{~F}$ & $2.4 \mathrm{E}+02$ & $0.0 \mathrm{E}+00$ & $8.2 \mathrm{E}-03$ & $0 \mathrm{E}$ & $0.0 \mathrm{E}+00$ & $1.1 \mathrm{E}-01$ & $0.0 \mathrm{E}+00$ & $5.2 \mathrm{E}+00$ & $0.0 \mathrm{E}+00$ & $1.8 \mathrm{E}+04$ & $3.2 \mathrm{E}+03$ & $8.3 \mathrm{E}+02$ & & & $1.4 \mathrm{E}+03$ & $3.4 \mathrm{E}+01$ & $0.0 \mathrm{E}+00$ & $1.2 \mathrm{E}+04$ & $8.7 \mathrm{E}-08$ \\
\hline $19 \mathrm{~F}$ & $9.4 \mathrm{E}-01$ & $0.0 \mathrm{E}+00$ & $2.8 \mathrm{E}-05$ & $0.0 \mathrm{E}+00$ & $0.0 \mathrm{E}$ & & $0.0 \mathrm{E}$ & $1.8 \mathrm{E}-02$ & $0.0 \mathrm{E}+00$ & $7.6 \mathrm{E}+01$ & 0.1 .1 & $\frac{0.0 \mathrm{~L}}{2.4 \mathrm{E}}$ & $2.0 \mathrm{E}$ & & $3.4 \mathrm{E}+01$ & $0.0 \mathrm{E}+00$ & $0.0 \mathrm{E}+00$ & $3.0 \mathrm{E}-04$ & $2.9 \mathrm{E}-10$ \\
\hline 26 & $6 \mathrm{E}-1$ & 0 & $3 \mathrm{E}-0$ & & & & & & 7. & & & & & & & & $0.0 \mathrm{E}+0$ & $\mathrm{E}-01$ & 7.6E-08 \\
\hline 331 & 3.6 & & 1. & & 2.1 & & 1.2 & 3.1 & 9.5 & & & & & & $9.4 \mathrm{E}+02$ & $2.4 \mathrm{E}+04$ & $2.2 \mathrm{E}+01$ & $6.4 \mathrm{E}+00$ & $3.5 \mathrm{E}-06$ \\
\hline $34 \mathrm{~F}$ & $2.6 \mathrm{E}+04$ & $0.0 \mathrm{E}+00$ & $1.1 \mathrm{E}$ & $0.0 \mathrm{E}$ & $0.0 \mathrm{E}$ & 1.11 & $0.0 \mathrm{E}$ & $8.1 \mathrm{E}+00$ & $6.2 \mathrm{E}+00$ & 0.0 & 1.3 & $2.9 \mathrm{E}$ & 4.3 & & $3.7 \mathrm{E}+02$ & $1.5 \mathrm{E}+05$ & $2.1 \mathrm{E}+01$ & $5.3 \mathrm{E}+00$ & 3.4E-06 \\
\hline $47 \mathrm{~F}$ & $4.5 \mathrm{E}+02$ & $0.0 \mathrm{E}+00$ & $\frac{1.1 \mathrm{~L}}{6.4 \mathrm{E}-03}$ & $0.0 \mathrm{E}+00$ & $0.0 \mathrm{E}+00$ & $\frac{1.15-01}{1.9 \mathrm{E}-02}$ & $0.0 \mathrm{E}+00$ & $1.5 \mathrm{E}+00$ & $0.0 \mathrm{E}+00$ & $1.6 \mathrm{E}+04$ & $2.3 \mathrm{E}+03$ & $5.1 \mathrm{E}+02$ & $7.1 \mathrm{E}+03$ & $1.0 \mathrm{E}-01$ & $6.6 \mathrm{E}+02$ & $0.0 \mathrm{E}+00$ & $0.0 \mathrm{E}+00$ & $9.3 \mathrm{E}-02$ & $\begin{array}{l}0.4 \mathrm{E}-08 \\
\end{array}$ \\
\hline Total C & $8.7 \mathrm{E}+04$ & $0.0 \mathrm{E}+00$ & $6.1 \mathrm{E}-01$ & $5.8 \mathrm{E}-07$ & $2.1 \mathrm{E}-02$ & $1.4 \mathrm{E}+00$ & $1.2 \mathrm{E}-01$ & $8.0 \mathrm{E}+01$ & $4.5 \mathrm{E}+01$ & $1.2 \mathrm{E}+05$ & $2.8 \mathrm{E}+04$ & $6.3 \mathrm{E}+03$ & $7.2 \mathrm{E}+04$ & $3.5 \mathrm{E}+00$ & $6.9 \mathrm{E}+03$ & $2.4 \mathrm{E}+05$ & $1.2 \mathrm{E}+02$ & $5.9 \mathrm{E}+04$ & $1.0 \mathrm{E}+00$ \\
\hline $\mathrm{Ci} / \mathrm{kg} \mathrm{SS}$ & $3.6 \mathrm{E}-02$ & $0.0 \mathrm{E}+00$ & $2.5 \mathrm{E}-07$ & $2.4 \mathrm{E}-13$ & $8.8 \mathrm{E}-09$ & $5.9 \mathrm{E}-07$ & $5.0 \mathrm{E}-08$ & $3.3 \mathrm{E}-05$ & $1.9 \mathrm{E}-05$ & $5.0 \mathrm{E}-02$ & $1.2 \mathrm{E}-02$ & $2.6 \mathrm{E}-03$ & $3.0 \mathrm{E}-02$ & $1.4 \mathrm{E}-06$ & $2.9 \mathrm{E}-03$ & $9.8 \mathrm{E}-02$ & $4.9 \mathrm{E}-05$ & $2.5 \mathrm{E}-02$ & $4.3 \mathrm{E}-07$ \\
\hline
\end{tabular}


Table 3-4. Estimated Curies of Radionuclides in Sludge Solids (SS) Received into the H Area Waste Tanks (Curies have been adjusted for decay)

\begin{tabular}{|c|c|c|c|c|c|c|c|c|c|c|c|c|c|c|c|c|c|c|c|}
\hline Tank \# & Ư⿱ & $\begin{array}{l}\text { o } \\
\dot{\delta}\end{array}$ & 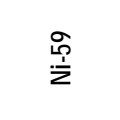 & $\begin{array}{l}\stackrel{R}{\dot{d}} \\
\stackrel{\infty}{\infty}\end{array}$ & 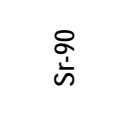 & $\underset{>}{\stackrel{\leftrightarrow}{>}}$ & 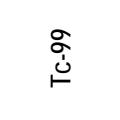 & $\begin{array}{l}\stackrel{\circ}{1} \\
\dot{\vec{\alpha}} \\
\end{array}$ & $\begin{array}{l}\stackrel{0}{T} \\
\frac{1}{\alpha}\end{array}$ & $\begin{array}{l}\text { đ̆ } \\
\text { ஸे }\end{array}$ & 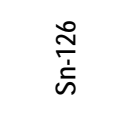 & స్తి & 岀 & 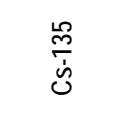 & 商 & 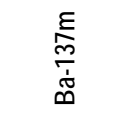 & $\begin{array}{l}\vec{J} \\
\dot{d}\end{array}$ & $\frac{\mathbb{J}}{\Delta}$ & \\
\hline $9 \mathrm{H}$ & $3.1 \mathrm{E}-02$ & $5.9 \mathrm{E}+01$ & $3.6 \mathrm{E}+01$ & 2.VLI I I & $6.5 \mathrm{E}+05$ & $6.5 \mathrm{E}+05$ & $\mathrm{E}+02$ & 3.0E-10 & $3.0 \mathrm{E}-10$ & $6.2 \mathrm{E}-01$ & $4.6 \mathrm{E}+01$ & $2.1 \mathrm{E}-03$ & $1.7 \mathrm{E}-04$ & $2.9 \mathrm{E}-01$ & $4.7 \mathrm{E}+04$ & $4.4 \mathrm{E}+04$ & $9.8 \mathrm{E}-15$ & $9.8 \mathrm{E}-15$ & $7.4 \mathrm{E}+00$ \\
\hline $10 \mathrm{H}$ & $3.2 \mathrm{E}-02$ & $6.6 \mathrm{E}+01$ & $3.7 \mathrm{E}+01$ & $2.5 \mathrm{E}+01$ & $6.7 \mathrm{E}+05$ & $6.7 \mathrm{E}+05$ & $4.4 \mathrm{E}+02$ & $5.6 \mathrm{E}-10$ & $5.6 \mathrm{E}-10$ & 7.7E-01 & $4.7 \mathrm{E}+01$ & $2.1 \mathrm{E}-03$ & $2.2 \mathrm{E}-04$ & 3.0E-01 & $4.9 \mathrm{E}+04$ & $4.6 \mathrm{E}+04$ & $2.4 \mathrm{E}-14$ & & $9.2 \mathrm{E}+00$ \\
\hline $11 \mathrm{H}$ & $2.5 \mathrm{E}-01$ & $4.9 \mathrm{E}+03$ & $2.8 \mathrm{E}+02$ & $1.5 \mathrm{E}+02$ & $6.8 \mathrm{E}+06$ & $6.8 \mathrm{E}+06$ & $2.6 \mathrm{E}+03$ & $2.1 \mathrm{E}-04$ & $2.1 \mathrm{E}-04$ & $7.7 \mathrm{E}+01$ & $1.4 \mathrm{E}+02$ & $8.9 \mathrm{E}-03$ & $1.9 \mathrm{E}+00$ & $1.7 \mathrm{E}+00$ & $3.8 \mathrm{E}+05$ & $3.6 \mathrm{E}+05$ & $.5 \mathrm{E}-06$ & $4.5 \mathrm{E}-06$ & $1.8 \mathrm{E}+03$ \\
\hline $12 \mathrm{H}$ & $2.5 \mathrm{E}-01$ & $3.7 \mathrm{E}+03$ & $2.8 \mathrm{E}+02$ & $1.6 \mathrm{E}+02$ & $6.6 \mathrm{E}+06$ & $6.6 \mathrm{E}+06$ & $2.7 \mathrm{E}+03$ & $9.6 \mathrm{E}-06$ & $9.6 \mathrm{E}-06$ & $4.0 \mathrm{E}+01$ & $1.6 \mathrm{E}+02$ & $9.6 \mathrm{E}-03$ & $6.9 \mathrm{E}-01$ & $1.8 \mathrm{E}+00$ & $3.8 \mathrm{E}+05$ & $3.5 \mathrm{E}+05$ & $4.0 \mathrm{E}-08$ & 4.0E-08 & $9.0 \mathrm{E}+02$ \\
\hline $13 \mathrm{H}$ & $4.3 \mathrm{E}-03$ & $6.9 \mathrm{E}+02$ & $3.4 \mathrm{E}+01$ & & & & $4.3 \mathrm{E}$ & & $4.5 \mathrm{E}-06$ & $1.1 \mathrm{E}$ & & 1.5 & & 2.9 & & & & & $2.5 \mathrm{E}+02$ \\
\hline $14 \mathrm{H}$ & $7.8 \mathrm{E}-03$ & $1.5 \mathrm{E}+02$ & $4.9 \mathrm{E}+01$ & $3.4 \mathrm{E}+01$ & $9.9 \mathrm{E}+05$ & $9.9 \mathrm{E}+05$ & $5.8 \mathrm{E}+02$ & 4.4E-09 & $4.4 \mathrm{E}-09$ & $1.7 \mathrm{E}+00$ & $5.8 \mathrm{E}+01$ & $2.6 \mathrm{E}-03$ & $2.6 \mathrm{E}-03$ & $3.9 \mathrm{E}-01$ & $6.8 \mathrm{E}+04$ & $6.4 \mathrm{E}+04$ & $1.9 \mathrm{E}-12$ & $1.9 \mathrm{E}-12$ & $2.2 \mathrm{E}+01$ \\
\hline $15 \mathrm{H}$ & $2.7 \mathrm{E}-01$ & $3.8 \mathrm{E}+03$ & $2.9 \mathrm{E}+02$ & $1.6 \mathrm{E}+02$ & $7.0 \mathrm{E}+06$ & $7.0 \mathrm{E}+06$ & $2.8 \mathrm{E}+03$ & $2.2 \mathrm{E}-05$ & $2.2 \mathrm{E}-05$ & $3.7 \mathrm{E}+01$ & $1.5 \mathrm{E}+02$ & $9.5 \mathrm{E}-03$ & $6.3 \mathrm{E}-01$ & $1.8 \mathrm{E}+00$ & $3.9 \mathrm{E}+05$ & $3.7 \mathrm{E}+05$ & $4.1 \mathrm{E}-07$ & $4.1 \mathrm{E}-07$ & $8.3 \mathrm{E}+02$ \\
\hline $16 \mathrm{H}$ & $6.5 \mathrm{E}-02$ & $3.5 \mathrm{E}+02$ & $7.4 \mathrm{E}+01$ & $4.2 \mathrm{E}+01$ & $1.5 \mathrm{E}+06$ & $1.5 \mathrm{E}+06$ & $7.0 \mathrm{E}+02$ & $1.8 \mathrm{E}-08$ & $1.8 \mathrm{E}-08$ & $1.3 \mathrm{E}+00$ & $3.8 \mathrm{E}+01$ & $2.4 \mathrm{E}-03$ & $1.1 \mathrm{E}-02$ & $4.7 \mathrm{E}-01$ & $8.4 \mathrm{E}+04$ & $8.0 \mathrm{E}+04$ & 3.0E-11 & 3.0E-11 & $2.6 \mathrm{E}+01$ \\
\hline $21 \mathrm{H}$ & $\mathrm{E}-04$ & & $5.2 \mathrm{E}$ & & $.3 \mathrm{E}$ & $2.3 \mathrm{E}$ & $6.8 \mathrm{E}$ & $1.3 \mathrm{E}$ & $1.3 \mathrm{E}-04$ & $1.4 \mathrm{~L}$ & & & & & & & & & $3.4 \mathrm{E}+02$ \\
\hline $22 \mathrm{H}$ & $0.0 \mathrm{E}+00$ & $4.4 \mathrm{E}+02$ & $8.8 \mathrm{E}+00$ & $6.8 \mathrm{E}+00$ & $3.7 \mathrm{E}+05$ & $3.7 \mathrm{E}+05$ & $1.2 \mathrm{E}+02$ & $9.3 \mathrm{E}-05$ & $9.3 \mathrm{E}-05$ & $1.6 \mathrm{E}+01$ & $6.3 \mathrm{E}$ & $4.0 \mathrm{E}-04$ & $5.2 \mathrm{E}-01$ & 7.7E-02 & $2.0 \mathrm{E}+04$ & $1.9 \mathrm{E}+04$ & $1.8 \mathrm{E}-06$ & $1.8 \mathrm{E}-06$ & $3.8 \mathrm{E}+02$ \\
\hline $30 \mathrm{H}$ & $7.8 \mathrm{E}-04$ & $1.2 \mathrm{E}+02$ & $8.6 \mathrm{E}$ & & $3.2 \mathrm{E}$ & $3.2 \mathrm{E}$ & $8.0 \mathrm{E}$ & $1.4 \mathrm{E}-03$ & $1.4 \mathrm{E}-03$ & & & $2.8 \mathrm{E}-05$ & 6.4 & $5.3 \mathrm{E}-03$ & & & & & $2.6 \mathrm{E}+02$ \\
\hline $32 \mathrm{H}$ & $2.4 \mathrm{E}-01$ & $1.4 \mathrm{E}+04$ & $2.7 \mathrm{E}+02$ & $1.5 \mathrm{E}+02$ & $8.1 \mathrm{E}+06$ & $8.1 \mathrm{E}+06$ & $2.5 \mathrm{E}+03$ & $1.1 \mathrm{E}-01$ & $1.1 \mathrm{E}-01$ & $7.2 \mathrm{E}+02$ & $1.4 \mathrm{E}$ & $8.7 \mathrm{E}-03$ & $4.2 \mathrm{E}+01$ & $1.7 \mathrm{E}+00$ & $4.5 \mathrm{E}+05$ & $4.2 \mathrm{E}+05$ & $1.1 \mathrm{E}-02$ & $1.1 \mathrm{E}-02$ & $1.9 \mathrm{E}+04$ \\
\hline $35 \mathrm{H}$ & $2.0 \mathrm{E}-01$ & $1.6 \mathrm{E}+04$ & $2.2 \mathrm{E}$ & $1.2 \mathrm{E}+02$ & $7.2 \mathrm{E}+06$ & $7.2 \mathrm{E}$ & $2.1 \mathrm{E}$ & & 1.3 & $8.3 \mathrm{E}+02$ & 1.1 & $7.2 \mathrm{E}-03$ & $4.6 \mathrm{E}$ & & & $3.8 \mathrm{E}+05$ & $1.5 \mathrm{E}-02$ & & $2.2 \mathrm{E}+04$ \\
\hline $36 \mathrm{H}$ & $2.3 \mathrm{E}-04$ & $1.3 \mathrm{E}+01$ & $2.6 \mathrm{E}$ & & $7.9 \mathrm{E}$ & $7.9 \mathrm{E}+03$ & $2.4 \mathrm{E}$ & $2.1 \mathrm{E}-06$ & $2.1 \mathrm{E}-06$ & & & $8.3 \mathrm{E}-06$ & $1.4 \mathrm{E}-02$ & 1.6 & & $4.1 \mathrm{E}+02$ & $3.1 \mathrm{E}-08$ & $3.1 \mathrm{E}-08$ & $1.0 \mathrm{E}+01$ \\
\hline $39 \mathrm{H}$ & $2.4 \mathrm{E}-01$ & $5.1 \mathrm{E}+04$ & $2.7 \mathrm{E}+02$ & $1.5 \mathrm{E}+02$ & $1.0 \mathrm{E}+07$ & $1.0 \mathrm{E}+07$ & $2.5 \mathrm{E}+03$ & $3.5 \mathrm{E}+01$ & $3.5 \mathrm{E}+01$ & $7.6 \mathrm{E}+03$ & $1.3 \mathrm{E}$ & $8.6 \mathrm{E}-03$ & $8.5 \mathrm{E}+02$ & $1.7 \mathrm{E}+00$ & $5.6 \mathrm{E}+05$ & $5.3 \mathrm{E}+05$ & $2.2 \mathrm{E}+01$ & $2.2 \mathrm{E}+01$ & $2.2 \mathrm{E}+05$ \\
\hline $41 \mathrm{H}$ & $0.0 \mathrm{E}+00$ & $1.8 \mathrm{E}+01$ & $2.1 \mathrm{E}-01$ & $1.6 \mathrm{E}-01$ & $9.8 \mathrm{E}+03$ & $9.8 \mathrm{E}+03$ & $2.7 \mathrm{E}+00$ & $2.3 \mathrm{E}-05$ & $2.3 \mathrm{E}-05$ & $1.1 \mathrm{E}+00$ & $1.5 \mathrm{E}-01$ & $9.4 \mathrm{E}-06$ & $4.9 \mathrm{E}-02$ & $1.8 \mathrm{E}-03$ & $5.4 \mathrm{E}+02$ & $5.1 \mathrm{E}+02$ & $7.0 \mathrm{E}-07$ & $7.0 \mathrm{E}-07$ & $2.8 \mathrm{E}+01$ \\
\hline $43 \mathrm{H}$ & $2.0 \mathrm{E}-02$ & $7.5 \mathrm{E}+03$ & $3.4 \mathrm{E}+01$ & $2.2 \mathrm{E}+01$ & $1.6 \mathrm{E}+06$ & $1.6 \mathrm{E}+06$ & $3.7 \mathrm{E}+02$ & $8.9 \mathrm{E}-01$ & $8.9 \mathrm{E}-01$ & $9.7 \mathrm{E}+02$ & $2.0 \mathrm{E}+01$ & $1.3 \mathrm{E}-03$ & $8.6 \mathrm{E}+01$ & $2.5 \mathrm{E}-01$ & $8.4 \mathrm{E}+04$ & $8.0 \mathrm{E}+04$ & $1.9 \mathrm{E}-01$ & $1.9 \mathrm{E}-01$ & $2.7 \mathrm{E}+04$ \\
\hline Total $\mathrm{Ci}$ & $1.6 \mathrm{E}+00$ & $1.0 \mathrm{E}+05$ & $1.9 \mathrm{E}+03$ & $1.1 \mathrm{E}+03$ & $5.3 \mathrm{E}+07$ & $5.3 \mathrm{E}+07$ & $1.8 \mathrm{E}+04$ & $3.7 \mathrm{E}+01$ & $3.7 \mathrm{E}+01$ & $1.0 \mathrm{E}+04$ & $1.1 \mathrm{E}+03$ & $6.5 \mathrm{E}-02$ & $1.0 \mathrm{E}+03$ & $1.2 \mathrm{E}+01$ & $3.0 \mathrm{E}+06$ & $2.8 \mathrm{E}+06$ & $2.3 \mathrm{E}+01$ & $2.3 \mathrm{E}+01$ & $2.9 \mathrm{E}+05$ \\
\hline Ci $/ \mathrm{kg}$ SS & $9.6 \mathrm{E}-07$ & $6.1 \mathrm{E}-02$ & $1.1 \mathrm{E}-03$ & $6.4 \mathrm{E}-04$ & $3.2 \mathrm{E}+01$ & $3.2 \mathrm{E}+01$ & $1.1 \mathrm{E}-02$ & $2.2 \mathrm{E}-05$ & $2.2 \mathrm{E}-05$ & $6.2 \mathrm{E}-03$ & $6.4 \mathrm{E}-04$ & $3.9 \mathrm{E}-08$ & $6.1 \mathrm{E}-04$ & 7.3E-06 & $1.8 \mathrm{E}+00$ & $1.7 \mathrm{E}+00$ & $1.4 \mathrm{E}-05$ & $1.4 \mathrm{E}-05$ & $1.7 \mathrm{E}-01$ \\
\hline
\end{tabular}

\begin{tabular}{|c|c|c|c|c|c|c|c|c|c|c|c|c|c|c|c|c|c|c|c|}
\hline Tank \# & 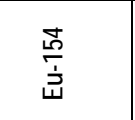 & 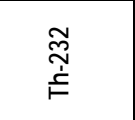 & $\begin{array}{l}\text { ָ̃ } \\
\text { J }\end{array}$ & 芯 & 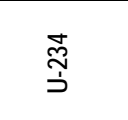 & 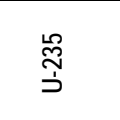 & $\begin{array}{l}\stackrel{\mathscr{N}}{J} \\
\text { J }\end{array}$ & 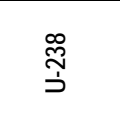 & 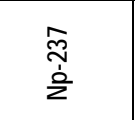 & 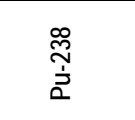 & $\begin{array}{c}\stackrel{8}{\dddot{乛}} \\
\stackrel{\bar{\alpha}}{0}\end{array}$ & 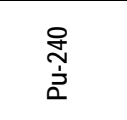 & 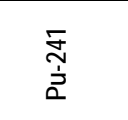 & $\begin{array}{l}\text { ָี } \\
\stackrel{\bar{\Xi}}{0}\end{array}$ & 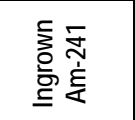 & $\underset{\text { 妾 }}{\vec{*}}$ & 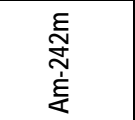 & 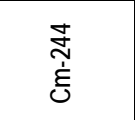 & $\begin{array}{l}\text { \&્ષ } \\
\text { Ė }\end{array}$ \\
\hline $9 \mathrm{HH}$ & $5 \mathrm{E}+02$ & $\overline{\mathrm{DE}+00}$ & $1.7 \mathrm{E}-02$ & $E+00$ & 0. & $1.2 \mathrm{E}-02$ & $0.0 \mathrm{E}+00$ & $2.9 \mathrm{E}-$ & $2.0 \mathrm{E}+$ & $4.4 \mathrm{E}+\mathrm{C}$ & $2 \mathrm{E}+01$ & $4 \mathrm{E}+01$ & $4.2 \mathrm{E}+01$ & $2.9 \mathrm{E}-03$ & $2.0 \mathrm{E}+01$ & $1 \mathrm{E}+03$ & $8 \mathrm{E}+00$ & $3.7 \mathrm{E}-01$ & $7.1 \mathrm{E}-07$ \\
\hline $10 \mathrm{H}$ & 02 & $=+00$ & $1.8 \mathrm{E}-02$ & $E+00$ & $\mathrm{E}+00$ & $2.3 \mathrm{E}-02$ & $\mathrm{E}+00$ & $5.7 \mathrm{E}-01$ & -00 & $2.2 \mathrm{E}+03$ & $2 \mathrm{E}+02$ & $7.1 \mathrm{E}+01$ & $2.3 \mathrm{E}+02$ & $1.5 \mathrm{E}-02$ & $1.0 \mathrm{E}+02$ & $3.2 \mathrm{E}+03$ & $3.9 \mathrm{E}+00$ & $3.9 \mathrm{E}-01$ & $7.2 \mathrm{E}-07$ \\
\hline $11 \mathrm{H}$ & $E+04$ & $6.0 \mathrm{E}-02$ & $0.0 \mathrm{E}+00$ & $8.5 \mathrm{E}+00$ & $3.2 \mathrm{E}+00$ & E-02 & $4.3 \mathrm{E}-01$ & $2.4 \mathrm{E}-01$ & $1.8 \mathrm{E}+00$ & $1.8 \mathrm{E}+05$ & $2.2 \mathrm{E}+03$ & $1.3 \mathrm{E}+03$ & $4.5 \mathrm{E}+04$ & $2.3 \mathrm{E}+00$ & $8.8 \mathrm{E}+03$ & $1.2 \mathrm{E}+04$ & $1.4 \mathrm{E}+01$ & $3.4 \mathrm{E}+03$ & $9.2 \mathrm{E}-03$ \\
\hline $12 \mathrm{H}$ & $3.4 \mathrm{E}+04$ & $1.4 \mathrm{E}+00$ & $1.3 \mathrm{E}-02$ & $3.8 \mathrm{E}+01$ & $3.6 \mathrm{E}+00$ & $6.7 \mathrm{E}-02$ & $2.8 \mathrm{E}-01$ & $6.8 \mathrm{E}-01$ & $7.9 \mathrm{E}+00$ & $1.3 \mathrm{E}+05$ & $2.3 \mathrm{E}+03$ & $1.3 \mathrm{E}+03$ & $2.3 \mathrm{E}+04$ & $1.8 \mathrm{E}+00$ & $4.9 \mathrm{E}+03$ & $1.3 \mathrm{E}+04$ & $1.5 \mathrm{E}+01$ & $2.1 \mathrm{E}+03$ & $8.3 \mathrm{E}-03$ \\
\hline $13 \mathrm{H}$ & $7.0 \mathrm{E}+03$ & $9.3 \mathrm{E}-\mathrm{C}$ & $0.0 \mathrm{E}+00$ & $3.2 \mathrm{E}+01$ & $3.9 \mathrm{E}+00$ & $6.4 \mathrm{E}-02$ & $4.0 \mathrm{E}-01$ & $3.8 \mathrm{E}$ & $4.8 \mathrm{E}+00$ & $1.1 \mathrm{E}+04$ & $6.8 \mathrm{E}+01$ & $2.3 \mathrm{E}+01$ & $3.7 \mathrm{E}+02$ & $2.2 \mathrm{E}-02$ & $7.1 \mathrm{E}+01$ & $1.5 \mathrm{E}+03$ & $1.7 \mathrm{E}+00$ & $8.9 \mathrm{E}+00$ & $1.5 \mathrm{E}-03$ \\
\hline $14 \mathrm{H}$ & $5 \mathrm{E}+03$ & $7.1 \mathrm{E}-02$ & $2.0 \mathrm{E}-02$ & $2.5 \mathrm{E}+00$ & $3.4 \mathrm{E}-01$ & $2.8 \mathrm{E}-02$ & $2.9 \mathrm{E}-02$ & $5.3 \mathrm{E}-01$ & $3.7 \mathrm{E}+00$ & $1.5 \mathrm{E}+03$ & $4.0 \mathrm{E}+02$ & $1.3 \mathrm{E}+02$ & $4.7 \mathrm{E}+02$ & $2.4 \mathrm{E}-02$ & $1.7 \mathrm{E}+02$ & $4.0 \mathrm{E}+03$ & $4.9 \mathrm{E}+00$ & $1.8 \mathrm{E}+00$ & $3.3 \mathrm{E}-04$ \\
\hline $15 \mathrm{H}$ & $E+04$ & $1.7 \mathrm{E}+00$ & $0.0 \mathrm{E}+00$ & $2.0 \mathrm{E}+01$ & $4.3 \mathrm{E}+00$ & $6.2 \mathrm{E}-02$ & $4.5 \mathrm{E}-01$ & $1.7 \mathrm{E}-03$ & $1.1 \mathrm{E}+00$ & $6.9 \mathrm{E}+04$ & $1.1 \mathrm{E}+03$ & $4.9 \mathrm{E}+02$ & $9.1 \mathrm{E}+03$ & 6.1 & $2.5 \mathrm{E}+03$ & $1.3 \mathrm{E}+04$ & $1.4 \mathrm{E}+01$ & $1.3 \mathrm{E}+03$ & $9.8 \mathrm{E}-03$ \\
\hline $16 \mathrm{H}$ & $51 \mathrm{~F}+03$ & $4.9 \mathrm{E}-03$ & $0.0 \mathrm{E}+00$ & $8.4 \mathrm{E}-02$ & $1.3 \mathrm{E}+00$ & $2.9 \mathrm{E}-02$ & $7.5 \mathrm{E}-02$ & $4.1 \mathrm{E}$ & $4.2 \mathrm{E}+00$ & $1.1 \mathrm{E}+04$ & $7.4 \mathrm{E}$ & $3.8 \mathrm{E}$ & & & $1.5 \mathrm{E}+03$ & $3.1 \mathrm{E}+03$ & $3.5 \mathrm{E}+00$ & $1.0 \mathrm{E}+01$ & $2.5 \mathrm{E}-03$ \\
\hline $21 \mathrm{r}$ & $2.2 \mathrm{E}+03$ & $0.0 \mathrm{E}+00$ & $0.0 \mathrm{E}+00$ & $0.0 \mathrm{E}+00$ & $9.3 \mathrm{E}-01$ & $1.2 \mathrm{E}-02$ & $2.1 \mathrm{E}-01$ & $1.8 \mathrm{E}$ & $5.4 \mathrm{E}-01$ & $5.4 \mathrm{E}+03$ & $2.1 \mathrm{E}+00$ & $1.7 \mathrm{E}+00$ & $9.1 \mathrm{E}+01$ & $4.2 \mathrm{E}-03$ & $1.2 \mathrm{E}+01$ & $2.3 \mathrm{E}+02$ & $2.7 \mathrm{E}-01$ & $2.0 \mathrm{E}+00$ & $2.4 \mathrm{E}-04$ \\
\hline 22 & $3.2 \mathrm{E}+03$ & $0.0 \mathrm{E}+00$ & $0.0 \mathrm{E}+00$ & $4.4 \mathrm{E}+00$ & $1.6 \mathrm{E}+00$ & $2.4 \mathrm{E}-02$ & $2.6 \mathrm{E}-01$ & $3.6 \mathrm{E}-02$ & \begin{tabular}{|c|}
$9.7 \mathrm{E}-01$ \\
\end{tabular} & $6.2 \mathrm{E}+03$ & $0.0 \mathrm{E}+00$ & $0.0 \mathrm{E}+00$ & $0.0 \mathrm{E}+00$ & $0.0 \mathrm{E}+00$ & $0.0 \mathrm{E}+00$ & $3.8 \mathrm{E}+02$ & $4.5 \mathrm{E}-01$ & $3.1 \mathrm{E}+00$ & $4.1 \mathrm{E}-04$ \\
\hline 30 & & $\mathrm{DE}+00$ & $0.0 \mathrm{E}+00$ & $0.0 \mathrm{E}+$ & $1.8 \mathrm{E}-02$ & -04 & $3.4 \mathrm{E}-03$ & $1.2 \mathrm{E}-05$ & $8.2 \mathrm{E}-03$ & $3.3 \mathrm{E}+03$ & $3.1 \mathrm{E}+01$ & $2.2 \mathrm{E}$ & $1.6 \mathrm{E}+03$ & $5.3 \mathrm{E}-02$ & $1.3 \mathrm{E}+02$ & $3.8 \mathrm{E}+01$ & $4.5 \mathrm{E}-02$ & $3.1 \mathrm{E}-01$ & $2.8 \mathrm{E}-05$ \\
\hline 32 & & & & & & & & & & & & & & & & & & & -03 \\
\hline 351 & & 10 & 0 & & $2.7 \mathrm{E}$ & $J \angle$ & $8.0 \mathrm{E}$ & 2.8 & $9.8 \mathrm{E}-01$ & 3.9 & 3.3 & & & $5.7 \mathrm{E}$ & 1.6E & 9.7 & $1.2 \mathrm{E}+01$ & $6.4 \mathrm{E}+01$ & 7.4E-03 \\
\hline & & 0. & $0.0 \mathrm{E}$ & & $7.3 \mathrm{E}$ & & 2.6 & $4.6 \mathrm{E}$ & $2.0 \mathrm{E}$ & $5.9 \mathrm{E}-$ & $4.9 \mathrm{E}$ & & 1.8 & 8.4 & $2.4 \mathrm{E}+$ & $1.1 \mathrm{E}+01$ & $1.3 \mathrm{E}-02$ & $6.8 \mathrm{E}-02$ & $8.5 \mathrm{E}-06$ \\
\hline 39 & 1. & 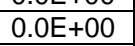 & $0.0 \mathrm{E}$ & $0.0 \mathrm{E}$ & $2.2 \mathrm{E}$ & & $3.7 \mathrm{E}$ & 4.0 & $1.3 \mathrm{E}$ & $6.0 \mathrm{E}$ & $7.9 \mathrm{E}$ & & 4.1 & & $2.6 \mathrm{E}+$ & $1.2 \mathrm{E}+04$ & $1.4 \mathrm{E}+01$ & $1.6 \mathrm{E}+05$ & $1.8 \mathrm{E}+01$ \\
\hline 4 & & & & & & & & & & & & & & & & & & & $9.7 \mathrm{E}-06$ \\
\hline & & $0.0 \mathrm{E}+00$ & $0.0 \mathrm{E}+00$ & & $3.4 \mathrm{E}+00$ & $4.7 \mathrm{E}-02$ & $6.5 \mathrm{E}-01$ & $3.8 \mathrm{E}-03$ & $3.6 \mathrm{E}+00$ & $7.8 \mathrm{E}+04$ & & $2.8 \mathrm{E}+02$ & $5.1 \mathrm{E}+04$ & $4.1 \mathrm{E}+00$ & $1.7 \mathrm{E}+03$ & $1.5 \mathrm{E}+03$ & $1.8 \mathrm{E}+00$ & $1.5 \mathrm{E}+01$ & $1.3 \mathrm{E}-03$ \\
\hline Total Ci & $4.6 \mathrm{E}+05$ & $3.3 \mathrm{E}+00$ & $6.7 \mathrm{E}-02$ & $1.1 \mathrm{E}+02$ & $5.0 \mathrm{E}+01$ & $8.6 \mathrm{E}-01$ & $8.0 \mathrm{E}+00$ & $2.8 \mathrm{E}+00$ & $5.0 \mathrm{E}+01$ & $1.9 \mathrm{E}+06$ & $2.2 \mathrm{E}+04$ & $1.4 \mathrm{E}+04$ & $8.1 \mathrm{E}+05$ & $3.1 \mathrm{E}+01$ & $8.0 \mathrm{E}+04$ & $8.8 \mathrm{E}+04$ & $1.0 \mathrm{E}+02$ & $1.7 \mathrm{E}+05$ & $1.8 \mathrm{E}+01$ \\
\hline $\mathrm{Ci} / \mathrm{kg} \mathrm{SS}$ & $2.8 \mathrm{E}-01$ & $1.9 \mathrm{E}-06$ & $4.0 \mathrm{E}-08$ & $6.3 \mathrm{E}-05$ & $3.0 \mathrm{E}-05$ & $5.1 \mathrm{E}-07$ & $4.7 \mathrm{E}-06$ & $1.7 \mathrm{E}-06$ & $3.0 \mathrm{E}-05$ & $1.1 \mathrm{E}+00$ & $1.3 \mathrm{E}-02$ & $8.3 \mathrm{E}-03$ & $4.8 \mathrm{E}-01$ & $1.8 \mathrm{E}-05$ & $4.7 \mathrm{E}-02$ & $5.2 \mathrm{E}-02$ & $6.1 \mathrm{E}-05$ & $1.0 \mathrm{E}-01$ & $1.0 \mathrm{E}-05$ \\
\hline
\end{tabular}


Also included in Tables 3-3 and 3-4 are: a) the total activity of each radionuclide received into the Tank Farm (second to last row); and b) the concentration of each radionuclide in sludge solids, averaged over the entire Tank Farm (last row).

The values in Tables 3-1 to 3-4 provide a basis for identifying chemical and radiological characteristics of the average sludge solids in the F and $\mathrm{H}$ Area tanks. As such, they can be used to identify general differences and trends between typical FTF sludge solids and typical HTF sludge solids. Similarly, they provide an indication of general differences to be expected between residual solids remaining in the F and $\mathrm{H}$ Tank Farms following tank cleaning and closure. However, due to wide variations in the canyon waste streams and the inherent heterogeneity of sludge in any given waste storage tank, the values in Tables 3-1 to 3-4 should not be considered representative of individual tank waste samples. Clearly, the composition of solids at any one location in a sludge tank could be significantly different from the composition at a different location. Still, the values in Tables 3-1 to 3-4 can provide insight into the relative dominance of constituents in $\mathrm{F}$ and $\mathrm{H}$ Area waste.

It should be noted that as sludge is dispositioned to the Defense Waste Processing Facility (DWPF), it is prepared in Tank $51 \mathrm{H}$, and then subsequently fed to DWPF from Tank 40H. As a consequence, when Tanks $51 \mathrm{H}$ and $40 \mathrm{H}$ are ultimately cleaned and closed, any waste residue remaining in these tanks will likely be a conglomeration of solids derived from both FTF and HTF source material. Although the impact of the FTF material will likely be small from the perspective of the entire HTF residual inventory, there is the expectation that the final residual material in Tanks $51 \mathrm{H}$ and $40 \mathrm{H}$ will be reflective of both FTF and HTF waste.

\subsubsection{Metals}

As shown in Tables 3-1 and 3-2, the total estimated mass of sludge solids received into the FTF was similar to that of the HTF, approximately two million kilograms. The most dominant metals at both Tank Farms were iron and aluminum, with iron hydroxide comprising $43 \%$ of the FTF sludge solids versus $34 \%$ of the HTF sludge solids, and aluminum hydroxide comprising $17 \%$ of the FTF sludge solids versus $32 \%$ of the HTF sludge solids. Given these differences, the iron to aluminum ratio for average FTF sludge is expected to be about two and a half times that of average HTF sludge. (Note that the typical iron to aluminum ratio of "HM only" sludge is lower than that of the average HTF sludge, which contains a combination of PUREX and HM sludge solids).

The third most dominant metal received into FTF was uranium, with uranyl hydroxide comprising $13 \%$ of the FTF sludge solids. In contrast, the uranyl hydroxide content of the HTF sludge solids is only about $0.7 \%$, a factor about twenty times lower than the FTF figure.

Other components of the FTF sludge contributing one or more percent of the solids included calcium carbonate $(\sim 5 \%)$, manganese dioxide $(\sim 5 \%)$, sodium hydroxide $(\sim 4 \%)$, nickel hydroxide $(\sim 3 \%)$, sodium chloride $(\sim 2 \%)$, silicon dioxide $(\sim 2 \%)$, and sodium 
nitrate $(\sim 1 \%)$. In contrast, other components of the HTF sludge contributing one or more percent include manganese dioxide $(\sim 6 \%)$, silicon dioxide $(\sim 5 \%)$, mercuric oxide $(\sim 4 \%)$, sodium nitrate $(\sim 4 \%)$, sodium hydroxide $(\sim 3 \%)$, calcium carbonate $(\sim 2 \%)$, calcium oxalate $(\sim 2 \%)$, and thorium dioxide $(\sim 2 \%)$. Omitting the readily soluble salts (sodium hydroxide, sodium chloride, and sodium nitrate) and the environmentally ubiquitous compounds (calcium carbonate and silicon dioxide), the most important differences include the greater dominance of nickel in FTF sludge, and the greater dominance of mercury, thorium, and oxalate in HTF sludge. The average nickel concentration in FTF sludge is about three times that of the HTF sludge, and the average mercury concentration in HTF sludge is about fifty times that of FTF. Per the receipt history, thorium and oxalate are expected to be absent from FTF sludge. However, given the recent use of oxalic acid as a chemical agent for cleaning Tanks $5 \mathrm{~F}$ and $6 \mathrm{~F}$, and the likely continued use of oxalic acid for future tank cleaning, there is the expectation that the oxalate content of the FTF waste is not zero and may increase over time.

Although present at low concentrations $(<1 \mathrm{wt} \%)$, the concentrations of silver and cobalt in FTF sludge are typically thirty to forty times those of the HTF sludge. Other low concentration metals that are more dominant in FTF sludge include: a) ruthenium, which is an average of ten times more concentrated at FTF; b) zinc, which is an average of six times more concentrated at FTF; and c) copper, which is an average of three times more concentrated at FTF.

\subsubsection{Radionuclides}

As shown in Tables 3-3 and 3-4, the radionuclides dominating the current sludge activity (CY 2012) include Sr-90/Y-90 and to a lesser degree, Cs-137/Ba-137m. This is the case at both FTF and HTF, due to the high fission yields of the mass 90 and 137 isotopes, and the relatively short half-lives of Sr-90 and Cs-137 (half-life $\approx 30$ years for both Sr-90 and Cs-137). The sludge phase activity of Sr-90 is higher than the sludge phase activity of Cs-137 (by an order of magnitude), because of the relatively low strontium solubility which partitions most strontium to the solid phase, as opposed to the relatively high cesium solubility which partitions most cesium to the liquid phase. The Sr-90 and Cs-137 concentrations of the average HTF sludge are two to three times those of the average FTF sludge, because of two primary factors: 1) the average HTF waste is "newer" than the average FTF waste (and therefore has been subjected to shorter periods of radiological decay); and 2) the burned enriched uranium processed at H Area typically underwent more fission reactions than the irradiated depleted uranium processed at $F$ Area.

With respect to radionuclide distributions, several differences exist between the average sludges at FTF and HTF. The uranium isotope distribution at FTF is consistent with that of depleted uranium, while the uranium isotope distribution at HTF is consistent with that of enriched uranium. At FTF, the ratio of $\mathrm{U}-235$ activity to $\mathrm{U}-238$ activity is about $2 \%$, equating to a mass ratio of about $0.3 \%$. In contrast, the activity ratio at HTF is about $30 \%$, equating to a mass ratio of about $5 \%$. Given that the U-235 content of natural uranium is $0.72 \mathrm{wt} \%$, it is clear that the average uranium at FTF is depleted and the 
average uranium at HTF is enriched. Additional indicators of the burned enriched uranium processed include the significantly higher activities of U-234 and U-236 received into HTF. (The mass of U-234 received into HTF is about 2000 times that of FTF, and the U-236 received into HTF is about 70 times that of FTF).

The plutonium isotope distribution at FTF is consistent with that of weapons grade plutonium, while the plutonium isotope distribution at HTF is consistent with that of higher burned plutonium and heat source plutonium. At FTF, the ratio of $\mathrm{Pu}-240$ activity to $\mathrm{Pu}-239$ activity is about $20 \%$, equating to a mass ratio of about $6 \%$ (typical of weapons grade plutonium). In contrast, the activity ratio at HTF is about $60 \%$, equating to a mass ratio of about $17 \%$, which is approximately three times that of average FTF material and indicative of higher burn-up. Consistent with the higher burn-up is the increased quantity of $\mathrm{Pu}-242$ received into $\mathrm{HTF}$, about an order of magnitude more than at FTF. Also apparent at HTF is the much higher $\mathrm{Pu}-238$ concentration, due to processing of heat source plutonium at $\mathrm{H}$ Area (the average $\mathrm{Pu}-238$ concentration in HTF sludge is about twenty times that of F Tank Farm sludge). Over the next several hundreds of thousands of years, the higher quantity of $\mathrm{Pu}-238$ will result in a commensurate increase in the quantities of long-term decay products Th-230 and Ra-226. As such, the long-term activities of Th-230 and Ra-226 at HTF will be about twenty times those of FTF. Note that in the shorter-term (over the next several hundred years), the Th-230 and Ra-226 activities at HTF are being driven by the Th-230 present as a Thoria impurity. ${ }^{13}$ This is in contrast to FTF, where both the short-term and long-term Th-230 and Ra-226 activities are driven by $\mathrm{Pu}-238$ decay.

Another difference is the presence of Th-232 and U-233 at HTF, due to the Thorex campaigns. Thorex campaign processing was limited to H Area, so Th-232 is expected to be absent from the FTF waste, and the quantity of U-233 at FTF is trivial compared to that of HTF (the U-233 activity received into FTF is $10^{-8}$ that of $\mathrm{H}$ Tank Farm). Th-232 comprises about two percent of the HTF sludge mass - however, its activity is relatively low $(\sim 3 \mathrm{Ci})$, due to the extremely long half-life $\left(\sim 10^{10}\right.$ years $)$. Given that the decay product of Th-232 is the relatively short-lived Ra-228 (half-life $\approx 6$ years), it is clear that the $\mathrm{Ra}-228$ activity will reach secular equilibrium with the Th-232 over the next couple of decades.

Also present in greater abundance in the HTF sludge are the quantities of the higher-mass actinide isotopes such as Cm-244 and Cm-245. At present, the concentrations of $\mathrm{Cm}-244$ and Cm-245 in HTF sludge are about four and twenty times those of FTF, respectively. However, because the Cm-244 half-life is relatively short ( $\sim 20$ years) and the Cm-245 half-life is relatively long ( 8500 years), only the differences of the $\mathrm{Cm}-245$ will persist into the long-term future (beyond a few hundred years).

\subsection{Evolution of Metal Compounds}

The metal compounds in the freshly received sludge (Tables 3-1 and 3-2) typically fall into one of a limited number of categories. Most transition metals, lanthanides, and uranium are assumed to be simple insoluble hydroxide compounds. Alkali metal salts are 
assumed to be soluble salts of nitrate, hydroxide, halides, sulfate, and/or phosphate. Alkaline earth metals and the heavy metal lead are assumed to exist as insoluble salts of carbonate, sulfate, phosphate, and/or oxalate. Mercury, manganese, ruthenium, silicon, and thorium are assumed to exist as oxides.

Over time, and with the advent of performing ancillary waste processing operations and chemical cleaning of tanks, the metal compounds are subject to change. For example, with the passage of time, many of the insoluble metal hydroxides are expected to transform to oxy-hydroxides and/or oxides, which are also insoluble. Absorption of atmospheric carbon dioxide will facilitate conversion to respective carbonate compounds, as applicable. Some of the metal compounds may react with other metal compounds to form complex structures containing multiple metals. Some of the metal compounds will remain stable over time and thus, unchanged. Understanding the potential for chemical changes to occur is important from the performance assessment perspective, since constituent solubility is a function of the chemical conditions.

Examples of chemical processing operations and tank cleaning agents that can potentially impact the chemistry of the solids are given below. Also given below are examples of alternative waste streams that have been introduced into the Tank Farms and can also impact chemical conditions.

\section{Waste Processing Operations}

- In-tank precipitation ${ }^{14}$ - use of tetraphenylborate to partition Cs to the solid phase

- Aluminum dissolution ${ }^{15-17}$ - caustic additions partitioning $\mathrm{Al}$ from solid-phase to liquid phase

\section{Chemical Cleaning Agents}

- Inhibited water ${ }^{18}$

- Oxalic acid solutions ${ }^{19,20}$

\section{Alternative Waste Streams}

- Cesium removal column (CRC)

- DWPF recycle

- Laboratory waste

- Potassium permanganate/nitric acid

- Salt dissolution

- Special discards

The potential impacts of ancillary processing operations and addition of alternative waste products can be significant or minimal, depending on the frequency of the operations and/or the quantity of materials that are introduced. The in-tank precipitation operations that were performed were limited to Tank $48 \mathrm{H}$. As a result, the characteristics of the 
Tank $48 \mathrm{H}$ solids are very different from those of other Tank Farm solids - the primary components of the Tank 48H insoluble solids are potassium, sodium, and cesium tetraphenylborate. Given the significant concentration of cesium, the Cs-137 activity content of the Tank $48 \mathrm{H}$ solids is significantly higher than that of typical sludge solids. Aluminum dissolution operations introduce high quantities of sodium hydroxide into sludge slurries, with the end result of converting solid phase aluminum in the sludge to liquid phase salt waste. Following aluminum dissolution, the aluminum content of the sludge is lower, the sodium content of the sludge is higher, and the iron to aluminum mass ratio of the sludge is correspondingly higher. Since aluminum in the form of gibbsite $\left(\mathrm{Al}(\mathrm{OH})_{3}\right)$ dissolves more rapidly than aluminum in the form of boehmite $(\mathrm{AlO}(\mathrm{OH}))$, the ratio of boehmite to gibbsite in post-aluminum dissolution sludge is higher than that of the original sludge.

Use of inhibited water as a tank cleaning agent following mechanical sludge removal has a relatively minor impact on the composition of residual solids. The low sodium hydroxide $(\mathrm{NaOH})$ and sodium nitrite $\left(\mathrm{NaNO}_{2}\right)$ content of the inhibited water tends to dilute the existing soluble sodium salts, and assuming the inhibited water solutions are removed after cleaning, leads to a reduced sodium content in the washed residual solids. Use of oxalic acid solution as a tank cleaning agent provides a source of oxalate anions which results in the formation of metal oxalates. At low $\mathrm{pH}$, the metal oxalates are typically soluble, which facilitates removal from the solid phase. Oxalic acid is particularly effective for dissolving iron, ${ }^{20}$ a primary component of sludge solids. Oxalic acid will also dissolve certain radioactive elements, including uranium. At high $\mathrm{pH}$, the metal oxalates are typically insoluble. As such, if oxalic acid cleaning waste is alkalized, metal oxalates will typically precipitate out of solution as a solid.

If Tank Farm supernatant solution is used as a solids transfer medium during the tank cleaning process, it could potentially introduce additional soluble waste constituents (primarily sodium salts and soluble radionuclides) into the residual solids matrix. The magnitude of the impact would be a function of the quantity of soluble waste constituents added. In cases where the residual solids were relatively free of soluble constituents, the impact could be significant.

Alternative waste streams received into the Tank Farms can also impact the composition of the solids. Receipt of cesium-sorbed zeolite solids from the cesium removal columns (CRCs) is a good example. Tanks that received such waste contain deposits of the sodium aluminosilicate zeolite matrix scattered amongst the traditional sludge solids. Given the function of the CRCs, it is expected that such zeolite deposits will be rich in Cs-137 compared to typical sludge solids. This phenomenon was demonstrated during characterization of residual waste samples collected following cleaning of Tank 19F, a tank that received CRC zeolite waste. The Cs-137/Ba-137m activities in the Tank 19F samples were orders of magnitude higher than the activities associated with any other radionuclides. ${ }^{38}$

The recycle stream from the DWPF is rich in silicates, due to the high silicon content introduced by the glass frit. When the DWPF recycle stream is received into the Tank 
Farm, there is the potential for the soluble-phase silicates to react with dissolved aluminum and form solid-phase sodium aluminosilicates, particularly when the resulting liquid waste stream is heated in the evaporator. A primary issue associated with such sodium aluminosilicate formation is accumulation of deposits of nitrated sodalite/nitrated cancrinite $\left(\mathrm{Na}_{8} \mathrm{Al}_{6} \mathrm{Si}_{6} \mathrm{O}_{24}\left(\mathrm{NO}_{3}\right)_{2} \cdot 4 \mathrm{H}_{2} \mathrm{O}\right)$ on the interior walls of the evaporator pot, and the corresponding difficulties associated with removing the deposits.

Receipt of laboratory waste into the Tank Farm is another operation which can impact the composition of tank waste, particularly in the area of radionuclide distributions.

Although the quantities of radionuclides utilized in the lab seem modest compared to those in the Tank Farms, they can still have significant impact in cases where the nuclides are typically minor constituents of the tank waste. An example is Pu-238 in FTF waste, where the contribution from laboratory waste is significant.

The combination of potassium permanganate and nitric acid, used as cleaning agent in various site operations, is another stream that affects waste composition when received into the Tank Farms. In addition to contributing potassium, magnesium, and nitrate to the waste, it can also enhance the redox conditions, due to the oxidizing qualities of the permanganate.

Other alternative streams (RBOF, 299-H maintenance, etc.) have been received into the Tank Farms, and each has the potential for affecting composition, depending on the relative quantities of waste involved and the constituent distributions. Each case must be evaluated individually to determine the significance of the impacts.

The form and composition of residual waste solids remaining following salt dissolution are expected to be significantly different from those of sludge solids. Whereas sludge solids typically contain high concentrations of iron compounds, solids remaining after salt dissolution have been characterized as containing high concentrations of other compounds, including gibbsite $\left(\mathrm{Al}(\mathrm{OH})_{3}\right)$ and burkeite $\left(\mathrm{Na}_{6} \mathrm{CO}_{3}\left(\mathrm{SO}_{4}\right)_{2}\right)$.

Salt waste processing activities may also impact the solids compositions. Carryover of solvent from the Caustic Side Solvent Extraction process could introduce constituents that are normally absent and/or alter certain solid-liquid phase partitioning behavior. Monosodium titanate used as a sorbent will be dispositioned to DWPF. However, monosodium titanate waste generated in the past was received into Tank $48 \mathrm{H}$, raising the titanium content of the Tank $48 \mathrm{H}$ waste.

Special discards of americium/curium, neptunium, and plutonium into the Tank Farm have been relatively limited to date and therefore have had a relatively minor impact on the total inventories of constituents. However, their impact with respect to any one affected tank is clearly greater. Addition of depleted uranium to Tank $41 \mathrm{H}$ is an example where the uranium isotopic distribution within an individual tank was altered significantly. 
A limited number of inter-area sludge transfers have occurred to date. Examples include transfers from Tanks $12 \mathrm{H}$ to $7 \mathrm{~F}, 11 \mathrm{H}$ to $8 \mathrm{~F}, 8 \mathrm{~F}$ to $21 \mathrm{H}$, and $18 \mathrm{~F}$ to $42 \mathrm{H}$. Such transfers introduce the potential for changing the compositions of FTF and HTF wastes, particularly if the frequency of the inter-area transfers increases in the future. Similarly, inter-area supernatant transfers could also change the constituent distributions, but will have clearly most impact on those constituents that are highly soluble (cesium, technetium, etc.).

\subsection{Mineral Phases in F and H Area Tank Farm Waste Solids}

A limited number of solids samples from the F and $\mathrm{H}$ Area waste tanks have been qualitatively analyzed for minerals via X-ray diffraction (XRD). ${ }^{20-26,36,37}$ The results of these analyses provide an indication of the dominant crystalline phases existing in the recent waste solids. A summary of the results is given in Table 3-5. As shown in the table, the samples were taken from various $\mathrm{F}$ and $\mathrm{H}$ Area waste tanks, and from a range of conditions - slurry solids collected during mechanical sludge removal; slurry solids collected following acid cleaning operations; precipitated solids from alkalized oxalic acid tank cleaning waste; residual solids following inhibited water cleaning operations; zeolite mound core solids remaining after bulk sludge removal; and slurry solids collected before and after aluminum dissolution processing.

Despite the range of conditions, there are many similarities between the minerals of the solids samples, and the dominant metals are consistent with expectations based on the relative metal abundance. Minerals of iron were identified in every sample, and minerals of aluminum, uranium, and sodium were identified in the majority of samples. Minerals of calcium and magnesium were identified in a smaller number of samples. Consistent with expectations, oxalate compounds were identified in the solids associated with oxalic acid cleaning operations.

The most common iron minerals were hematite $\left(\mathrm{Fe}_{2} \mathrm{O}_{3}\right)$ and magnetite $\left(\mathrm{Fe}_{3} \mathrm{O}_{4}\right)$, but maghemite $\left(\mathrm{Fe}_{2} \mathrm{O}_{3}\right)$, lepidocrocite $(\mathrm{FeO}(\mathrm{OH}))$, and goethite $(\mathrm{FeO}(\mathrm{OH}))$ were also identified. Aluminum was identified in three mineral forms - gibbsite $\left(\mathrm{Al}(\mathrm{OH})_{3}\right)$, boehmite $(\mathrm{AlO}(\mathrm{OH}))$, and nitrated cancrinite $\left(\mathrm{Na}_{8}\left(\mathrm{Al}_{6} \mathrm{Si}_{6} \mathrm{O}_{24}\right)\left(\mathrm{NO}_{3}\right)_{2} \cdot 4 \mathrm{H}_{2} \mathrm{O}\right)$. (Note that sodium and silicon are also components of the cancrinite). Uranium was identified in four mineral forms - clarkeite $\left(\mathrm{Na}\left(\left(\mathrm{UO}_{2}\right) \mathrm{O}\right)(\mathrm{OH}) \cdot \mathrm{H}_{2} \mathrm{O}\right)$, sodium uranium oxide hydrate $\left(\mathrm{Na}_{2} \mathrm{U}_{2} \mathrm{O}_{7} \cdot 6 \mathrm{H}_{2} \mathrm{O}\right)$, cejkaite $\left(\mathrm{Na}_{4} \mathrm{UO}_{2}\left(\mathrm{CO}_{3}\right)_{3}\right)$, and uranyl hydrogen fluoride hydrate $\left(\mathrm{UO}_{2} \mathrm{HF}_{3} \cdot 2 \mathrm{H}_{2} \mathrm{O}\right)$. Sodium was present in the cancrinite and the three sodium-uranium minerals identified above, as well as in natroxalate $\left(\mathrm{Na}_{2} \mathrm{C}_{2} \mathrm{O}_{4}\right)$, thermonatrite $\left(\mathrm{Na}_{2} \mathrm{CO}_{3} \cdot \mathrm{H}_{2} \mathrm{O}\right)$, and natratine $\left(\mathrm{NaNO}_{3}\right)$. The combination of calcium and magnesium was identified in one mineral form - dolomite $\left(\mathrm{CaMg}\left(\mathrm{CO}_{3}\right)_{2}\right)$ - and calcium alone was identified in calcite $\left(\mathrm{CaCO}_{3}\right)$. Given that strontium and radium are chemically similar to calcium and magnesium (due to being alkaline earth metals), there is the expectation that strontium and radium currently exist in mineral forms such as $\mathrm{XY}\left(\mathrm{CO}_{3}\right)_{2}$ and $\mathrm{XCO}_{3}$, where $\mathrm{X}=\mathrm{Sr}$ or $\mathrm{Ra}$, and $\mathrm{Y}=$ one of the alkaline earth metals. 
SRNL-STI-2012-00479

Revision 0

Table 3-5. Mineral Phases Identified in F and H Area Tank Farm Waste Solids

\begin{tabular}{|c|c|c|c|}
\hline Tank & Description & Mineral Phases & Reference \\
\hline $4 \mathrm{~F}$ & $\begin{array}{l}\text { Slurry solids obtained } \\
\text { during mechanical } \\
\text { sludge removal } \\
\text { operations }\end{array}$ & $\begin{array}{l}\text { Hematite }\left(\mathrm{Fe}_{2} \mathrm{O}_{3}\right) \\
\text { Magnetite }\left(\mathrm{Fe}_{3} \mathrm{O}_{4}\right) \\
\text { Clarkeite }\left(\mathrm{Na}\left(\left(\mathrm{UO}_{2}\right) \mathrm{O}\right)(\mathrm{OH}) \cdot \mathrm{H}_{2} \mathrm{O}\right)\end{array}$ & 20 \\
\hline $5 \mathrm{~F}$ & $\begin{array}{l}\text { Solids obtained } \\
\text { following oxalic acid } \\
\text { cleaning of Tank } 5 \mathrm{~F}\end{array}$ & $\begin{array}{l}\text { Hematite }\left(\mathrm{Fe}_{2} \mathrm{O}_{3}\right) \\
\text { Sodium cobalt oxalate }\left(\mathrm{Na}_{2} \mathrm{Co}\left(\mathrm{C}_{2} \mathrm{O}_{4}\right)_{2}\right) \\
\text { Magnetite }\left(\mathrm{Fe}_{3} \mathrm{O}_{4}\right) \\
\text { Nickel oxalate hydrate }\left(\mathrm{NiC}_{2} \mathrm{O}_{4} \cdot 2 \mathrm{H}_{2} \mathrm{O}\right)\end{array}$ & 36 \\
\hline $6 \mathrm{~F}$ & $\begin{array}{l}\text { Solids obtained } \\
\text { following oxalic acid } \\
\text { cleaning of Tank } 6 \mathrm{~F}\end{array}$ & $\begin{array}{l}\text { Hematite }\left(\mathrm{Fe}_{2} \mathrm{O}_{3}\right) \\
\text { Maghemite }\left(\mathrm{Fe}_{2} \mathrm{O}_{3}\right) \\
\text { Nickel oxalate hydrate }\left(\mathrm{NiC}_{2} \mathrm{O}_{4} \cdot 2 \mathrm{H}_{2} \mathrm{O}\right) \\
\text { Goethite }(\mathrm{FeO}(\mathrm{OH}))\end{array}$ & 37 \\
\hline $7 F$ & $\begin{array}{l}\text { Precipitated solids } \\
\text { generated through } \\
\text { alkalization of oxalic } \\
\text { acid cleaning solution } \\
\text { used for removing } \\
\text { residual solids from } \\
\text { Tanks 5F and 6F }\end{array}$ & $\begin{array}{l}\text { Magnetite }\left(\mathrm{Fe}_{3} \mathrm{O}_{4}\right) \\
\text { Clarkeite }\left(\mathrm{Na}\left(\left(\mathrm{UO}_{2}\right) \mathrm{O}\right)(\mathrm{OH}) \cdot \mathrm{H}_{2} \mathrm{O}\right. \\
\text { Nitrated cancrinite }\left(\mathrm{Na}_{8}\left(\mathrm{Al}_{6} \mathrm{Si}_{6} \mathrm{O}_{24}\right)\left(\mathrm{NO}_{3}\right)_{2} \cdot 4 \mathrm{H}_{2} \mathrm{O}\right) \\
\text { Natroxalate }\left(\mathrm{Na}_{2} \mathrm{C}_{2} \mathrm{O}_{4}\right) \\
\text { Sodium uranium oxide hydrate }\left(\mathrm{Na}_{2} \mathrm{U}_{2} \mathrm{O}_{7} \cdot 6 \mathrm{H}_{2} \mathrm{O}\right)\end{array}$ & 21 \\
\hline $12 \mathrm{H}$ & $\begin{array}{l}\text { Solids obtained during } \\
\text { mechanical sludge } \\
\text { removal operations }\end{array}$ & $\begin{array}{l}\text { Boehmite }(\mathrm{AlO}(\mathrm{OH})) \\
\text { Hematite }\left(\mathrm{Fe}_{2} \mathrm{O}_{3}\right) \\
\text { Dolomite }\left(\mathrm{CaMg}\left(\mathrm{CO}_{3}\right)_{2}\right) \\
\text { Thermonatrite }\left(\mathrm{Na}_{2} \mathrm{CO}_{3} \cdot \mathrm{H}_{2} \mathrm{O}\right)\end{array}$ & 22 \\
\hline $18 \mathrm{~F}$ & $\begin{array}{l}\text { Residual solids } \\
\text { obtained following } \\
\text { inhibited water } \\
\text { cleaning of Tank } 18 \mathrm{~F}\end{array}$ & $\begin{array}{l}\text { Cejkaite }\left(\mathrm{Na}_{4} \mathrm{UO}_{2}\left(\mathrm{CO}_{3}\right)_{3}\right) \\
\text { Gibbsite }\left(\mathrm{Al}(\mathrm{OH})_{3}\right) \\
\text { Uranyl hydrogen fluoride hydrate }\left(\mathrm{UO}_{2} \mathrm{HF}_{3} \cdot 2 \mathrm{H}_{2} \mathrm{O}\right) \\
\text { Nitrated cancrinite }\left(\mathrm{Na}_{8}\left(\mathrm{Al}_{6} \mathrm{Si}_{6} \mathrm{O}_{24}\right)\left(\mathrm{NO}_{3}\right)_{2} \cdot 4 \mathrm{H}_{2} \mathrm{O}\right) \\
\text { Hematite }\left(\mathrm{Fe}_{2} \mathrm{O}_{3}\right) \\
\text { Calcite }\left(\mathrm{CaCO}_{3}\right)\end{array}$ & 23 \\
\hline $19 \mathrm{~F}$ & $\begin{array}{l}\text { Zeolite mound core } \\
\text { solids obtained } \\
\text { following mechanical } \\
\text { bulk sludge removal }\end{array}$ & $\begin{array}{l}\text { Nitrated cancrinite }\left(\mathrm{Na}_{8}\left(\mathrm{Al}_{6} \mathrm{Si}_{6} \mathrm{O}_{24}\right)\left(\mathrm{NO}_{3}\right)_{2} \cdot 4 \mathrm{H}_{2} \mathrm{O}\right) \\
\text { Gibbsite }\left(\mathrm{Al}(\mathrm{OH})_{3}\right) \\
\text { Maghemite }\left(\mathrm{Fe}_{2} \mathrm{O}_{3}\right) \\
\text { Lepidocrocite }(\mathrm{FeO}(\mathrm{OH})) \\
\text { Goethite }(\mathrm{FeO}(\mathrm{OH}))\end{array}$ & 24 \\
\hline $42 \mathrm{H}$ & $\begin{array}{l}\text { Combined sludge slurry } \\
\text { from Tanks } 15 \mathrm{H} \& 18 \mathrm{~F}\end{array}$ & $\begin{array}{l}\text { Gibbsite }\left(\mathrm{Al}(\mathrm{OH})_{3}\right) \\
\text { Hematite }\left(\mathrm{Fe}_{2} \mathrm{O}_{3}\right)\end{array}$ & 25 \\
\hline $51 \mathrm{H}$ & $\begin{array}{l}\text { Sludge slurry solids } \\
\text { transferred from Tank } \\
11 \mathrm{H} \text {. Solids obtained } \\
\text { prior to aluminum } \\
\text { dissolution }\end{array}$ & $\begin{array}{l}\text { Boehmite }(\mathrm{AlO}(\mathrm{OH})) \\
\text { Hematite }\left(\mathrm{Fe}_{2} \mathrm{O}_{3}\right) \\
\text { Clarkeite }\left(\mathrm{Na}\left(\left(\mathrm{UO}_{2}\right) \mathrm{O}\right)(\mathrm{OH}) \cdot \mathrm{H}_{2} \mathrm{O}\right) \\
\text { Dolomite }\left(\mathrm{CaMg}\left(\mathrm{CO}_{3}\right)_{2}\right) \\
\text { Gibbsite }\left(\mathrm{Al}(\mathrm{OH})_{3}\right) \\
\text { Sodium uranium oxide hydrate }\left(\mathrm{Na}_{2} \mathrm{U}_{2} \mathrm{O}_{7} \cdot 6 \mathrm{H}_{2} \mathrm{O}\right)\end{array}$ & 26 \\
\hline $51 \mathrm{H}$ & $\begin{array}{l}\text { Sludge slurry solids } \\
\text { transferred from } 11 \mathrm{H} . \\
\text { Solids obtained after } \\
\text { aluminum dissolution }\end{array}$ & $\begin{array}{l}\text { Boehmite }(\mathrm{AlO}(\mathrm{OH})) \\
\text { Hematite }\left(\mathrm{Fe}_{2} \mathrm{O}_{3}\right) \\
\text { Clarkeite }\left(\mathrm{Na}\left(\left(\mathrm{UO}_{2}\right) \mathrm{O}\right)(\mathrm{OH}) \cdot \mathrm{H}_{2} \mathrm{O}\right) \\
\text { Thermonatrite }\left(\mathrm{Na}_{2} \mathrm{CO}_{3} \cdot \mathrm{H}_{2} \mathrm{O}\right) \\
\text { Natratine }\left(\mathrm{NaNO}_{3}\right) \\
\end{array}$ & 26 \\
\hline
\end{tabular}


A couple of trends are worth noting. Aluminum in the form of boehmite was only found in the solids originating solely from $\mathrm{H}$ Area (Tanks $11 \mathrm{H}$ and $12 \mathrm{H}$ ). Aluminum in the form of gibbsite was found in both the $\mathrm{F}$ and $\mathrm{H}$ Area tanks, as was aluminum in the form of nitrated cancrinite. Differences between the pre- and post-aluminum dissolution sludge of Tank $11 \mathrm{H}$ suggest that the aluminum dissolution process removed gibbsite and increased the quantities of sodium salts, which is consistent with expectations. A total of three oxalate-containing compounds (sodium cobalt oxalate, nickel oxalate hydrate, and natroxalate) were found in the solids that had been in contact with oxalic acid cleaning solution or had been precipitated from oxalic acid cleaning waste. Of the metals identified in the various minerals, uranium appeared to be the one present in the largest number of different chemical compounds.

\subsection{Solid/Liquid Phase Technetium Partitioning}

Tc-99 is a particularly important radionuclide because of its potential mobility in the environment and its long half-life ( $\sim 2 \mathrm{E}+05$ years). Technetium can exist in five different oxidation states, ${ }^{27}$ but the states most prevalent for environmental conditions are Tc(IV) and Tc(VII). ${ }^{28}$ In SRS Tank Farm waste, it is assumed that the vast majority of technetium existing in sludge solids is present as Tc(IV) and relatively insoluble, in the form of hydrated technetium dioxide $\left(\mathrm{TcO}_{2} \cdot \mathrm{xH}_{2} \mathrm{O}\right)^{29}$ and/or co-precipitated with iron(II) sorbed onto iron(III) oxide and/or oxyhydroxide particles. ${ }^{30,31}$ In contrast, the majority of technetium existing in salt waste is assumed to be present as $\mathrm{Tc}$ (VII) and relatively soluble, in the form of sodium pertechnetate $\left(\mathrm{NaTcO}_{4}\right){ }^{29}$ Some portion of the soluble technetium could also be present as a Tc(IV)-chelant complex ${ }^{32}$ or as a Tc(VII)-solvent complex, ${ }^{33}$ but these portions are expected to be minor in most SRS waste tanks due to the very low organic content.

The fraction of technetium that is readily soluble in Tank Farm waste is variable, based on the tank chemistry conditions and the processing operations that have been performed. Research activities at SRS and at Hanford have identified soluble technetium portions ranging from 20 to $95 \%$. Fowler et al. ${ }^{29}$ identified that about $60 \%$ of the technetium in the SRS Tank Farm is present in salt waste and highly soluble. Bibler et al. ${ }^{34}$ identified that up to about $70 \%$ of technetium in a simulated high level waste was soluble following alkalization and sludge washing. In contrast, real waste sludge slurry samples from the SRS Tank Farm were found to contain only 5-10\% of the total technetium, indicating $90-95 \%$ of the total technetium was soluble. Extensive washing of a real-waste sludge slurry, using inhibited water as the washing solution, indicated that the only technetium that could be "washed out" of the slurry was the portion already dissolved in the supernatant phase. This suggests that the portion of technetium in the sludge solids (Tc(IV)) was stable and not prone to oxidation during inhibited water washing. The portions of soluble technetium in two Hanford waste tanks varied significantly from one another, $20 \%$ in one waste tank and $80 \%$ in the other waste tank. ${ }^{35}$

Of greatest potential impact to the technetium solubility are: a) the relative amounts of iron(II) and iron(III) present during and after waste alkalization; b) the redox conditions of the alkalized waste; c) the availability of atmospheric oxygen and/or other oxidizing 
agents (which can oxidize $\mathrm{Tc}(\mathrm{IV})$ to $\mathrm{Tc}(\mathrm{VII})$; d) the kinetics for equilibrating the oxidizing agents with the alkalized waste; and e) the chemical, physical, and thermal processes impacting the final equilibration state and the kinetics of the equilibration process.

In assessing solid/liquid phase technetium partitioning, it is probably most useful to address the cases expected to drive the majority of Tank Farm technetium behavior. These cases include: 1) Tc(IV) present as $\mathrm{TcO}_{2}$ in sludge solids; 2) $\mathrm{Tc}(\mathrm{IV})$ coprecipitated with iron in sludge solids; 3 ) $\mathrm{Tc}(\mathrm{VII})$ present as soluble $\mathrm{TcO}_{4}{ }^{-}$in supernatant and/or saltcake; and d) the combination of Tc(IV) and Tc(VII) present in sludge solids and in supernatant/saltcake.

In the case of $\mathrm{Tc}(\mathrm{IV}) \mathrm{O}_{2}$, the technetium is expected to remain relatively insoluble unless one of the two following changes occur. The first is oxidation of the Tc(IV) to $\mathrm{Tc}(\mathrm{VII})$, facilitating formation of pertechnetate $\left(\mathrm{TcO}_{4}{ }^{-}\right)$(which is highly soluble). Such oxidation could occur over time via interaction with atmospheric oxygen, oxygen from nitrate radiolysis, and/or other oxidation agents. Alternatively, in the presence of a reducing agent, the technetium could remain relatively insoluble in the Tc(IV) state, assuming stability of the $\mathrm{TcO}_{2}$ form and absence of Tc(IV) complexing agents. Presence of an applicable technetium complexing agent provides an alternative means that relatively insoluble Te(IV) forms could be converted to a more soluble form.

In the case of $\mathrm{Tc}(\mathrm{IV})$ co-precipitated with solid-phase iron, the technetium is expected to remain relatively insoluble unless one of the following changes occur. The first is what was described above, oxidation of the Te(IV) to Te(VII), facilitating formation of pertechnetate (soluble). In order for this to occur, an oxidizing agent must be present and be given sufficient time to migrate to the solid phase sites where the technetium is located. Since a large portion of the technetium would be present within the interior of the iron mineral particles, the time necessary for the oxidizing agents to gain access to the technetium could be considerable. The second change that could lead to release of technetium would be dissolution of the iron minerals acting as the carrier. Once released from the carrier, the fate of the Tc(IV) would be driven by the prevailing redox conditions - if adequately oxidizing, the $\mathrm{Tc}(\mathrm{IV})$ would likely be converted to $\mathrm{Tc}(\mathrm{VII})$, facilitating formation of pertechnetate (soluble); if adequately reducing, the Tc(IV) would likely take a more insoluble form such as $\mathrm{TcO}_{2}$, and/or possibly become associated with other available solid phase particles via sorption. As in the first Tc(IV) case, presence of an applicable technetium complexing agent could provide a means for the normally low solubility Tc(IV) to become soluble.

In the case of $\mathrm{Tc}(\mathrm{VII})$ present as soluble $\mathrm{TcO}_{4}{ }^{-}$, the technetium is likely to retain its form assuming oxidizing conditions prevail. However, if sufficient reducing conditions prevail, the $\mathrm{Tc}(\mathrm{VII})$ would be reduced to $\mathrm{Tc}(\mathrm{IV})$, facilitating insolubility and sorption, assuming the absence of applicable technetium complexing agents. Of course, subsequent redox changes back to oxidizing conditions would facilitate conversion back to the more soluble $\mathrm{Tc}(\mathrm{VII})$ form. 
The combination of $\mathrm{Tc}(\mathrm{IV})$ and $\mathrm{Tc}(\mathrm{VII})$ is expected to be present in cases where sludge solids (or sludge solids residue) are mixed with soluble salt waste solids. An example is a sludge slurry, where $\mathrm{Tc}(\mathrm{IV})$ is present in the solid phase, and $\mathrm{Tc}(\mathrm{VII})$ is present in the supernatant phase (liquid phase). Another example is the combination of sludge solids (containing $\mathrm{Tc}(\mathrm{IV})$ ) and saltcake (containing $\mathrm{Tc}(\mathrm{VII})$ ). Under normal circumstances in such cases, it is assumed that both the Tc(IV) in the sludge solids and the $\mathrm{Tc}$ (VII) in the salt waste are relatively stable. However, with the addition of oxidizing agents, reducing agents, complexing agents, and/or organic solvents, the technetium oxidation states and associated partitioning would be prone to the same transitions addressed above.

\subsection{Conclusions}

1) Important differences between the projected average concentrations of metal compounds in F and $\mathrm{H}$ Area Tank Farm waste solids are summarized below:

\begin{tabular}{|l|c|}
\hline Metal Descriptor & Ratio of FTF value to HTF value \\
\hline \hline Iron to aluminum concentration ratio & $\sim 2$ \\
\hline Uranium concentration & $\sim 20$ \\
\hline Mercury concentration & $\sim 0.02$ \\
\hline Nickel concentration & $\sim 3$ \\
\hline Ruthenium concentration & $\sim 10$ \\
\hline Zinc concentration & $\sim 6$ \\
\hline Silver concentration & $\sim 30$ \\
\hline Cobalt concentration & $\sim 40$ \\
\hline Copper concentration & $\sim 3$ \\
\hline
\end{tabular}

2) Other differences between the compositions of FTF and HTF waste solids include:

- Uranium isotope distribution

o Depleted uranium at FTF $(\sim 0.3 \mathrm{wt} \% \mathrm{U}-235)$

0 Enriched uranium at $\operatorname{HTF}(\sim 5 \mathrm{wt} \% \mathrm{U}-235)$

o $\mathrm{U}-234$ at $\mathrm{HTF} \approx 2000 \mathrm{X}$ that at FTF

o U-236 at $\mathrm{HTF} \approx 70 \mathrm{X}$ that at FTF

- Plutonium and higher actinide isotope distributions

o Weapons grade plutonium at FTF $(\sim 6 \mathrm{wt} \% \mathrm{Pu}-240)$

o High burned plutonium at $\operatorname{HTF}(\sim 17 \mathrm{wt} \% \mathrm{Pu}-240)$

o $\mathrm{Pu}-238$ at $\mathrm{HTF} \approx 20 \mathrm{X}$ that at $\mathrm{FTF}$

- 20X more long-term Th-230 and Ra-226 at HTF

o $\mathrm{Pu}-242$ at $\mathrm{HTF} \approx 10 \mathrm{X}$ that at FTF

o $\mathrm{Cm}-244$ at $\mathrm{HTF} \approx 4 \mathrm{X}$ that at FTF

o $\mathrm{Cm}-245$ at $\mathrm{HTF} \approx 20 \mathrm{X}$ that at FTF

- Thoria processed solely at HTF

o Thorium dioxide $\approx 2 \mathrm{wt} \%$ of HTF solids

o U-233 at HTF $\approx 10^{8}$ times that at FTF

- Calcium oxalate $\approx 2 \mathrm{wt} \%$ of HTF solids 
3) Dominant crystalline components of the waste solids include:

- Iron oxide and oxyhydroxide compounds $\left(\mathrm{Fe}_{2} \mathrm{O}_{3}, \mathrm{Fe}_{3} \mathrm{O}_{4}, \mathrm{FeO}(\mathrm{OH})\right)$

- Aluminum hydroxide and oxyhydroxide compounds $\left(\mathrm{Al}(\mathrm{OH})_{3}, \mathrm{AlO}(\mathrm{OH})\right)$

- Various uranium compounds $\left(\mathrm{Na}\left(\left(\mathrm{UO}_{2}\right) \mathrm{O}\right)(\mathrm{OH}) \cdot \mathrm{H}_{2} \mathrm{O}, \mathrm{Na}_{2} \mathrm{U}_{2} \mathrm{O}_{7} \cdot 6 \mathrm{H}_{2} \mathrm{O}\right.$, $\left.\mathrm{Na}_{4} \mathrm{UO}_{2}\left(\mathrm{CO}_{3}\right)_{3}\right)$

- A nitrated sodium aluminosilicate compound $\left(\mathrm{Na}_{8}\left(\mathrm{Al}_{6} \mathrm{Si}_{6} \mathrm{O}_{24}\right)\left(\mathrm{NO}_{3}\right)_{2} \cdot 4 \mathrm{H}_{2} \mathrm{O}\right)$

- Carbonates of calcium, magnesium, and sodium $\left(\mathrm{CaMg}\left(\mathrm{CO}_{3}\right)_{2}, \mathrm{Na}_{2} \mathrm{CO}_{3} \cdot \mathrm{H}_{2} \mathrm{O}\right)$

- Oxalate compounds in the solids associated with oxalic acid cleaning

4) The primary oxidation state of technetium in SRS sludge solids is Tc(IV), existing in the form of technetium dioxide and/or co-precipitated with iron. In contrast, the primary oxidation state of technetium in SRS salt waste (supernatant and saltcake) is Tc(VII), existing in the form of sodium pertechnetate. On a relative basis, technetium dioxide and technetium co-precipitated with iron are considered very insoluble under typical waste conditions, as opposed to the sodium pertechnetate which is considered very soluble. Over time, with changes in redox chemistry, technetium solubilities are subject to change as oxidation state changes and/or other chemical condition changes occur. In the absence of reducing agents, the expectation is that technetium in the environment will eventually become oxidized (and therefore readily soluble), due to the high availability of atmospheric oxygen. However, the time requirements for this to happen will be variable, depending on the physical, chemical, and thermal conditions.

\subsection{References}

1 Chew, D. P., “SRS Waste Tank Charts," SRR-LWP-2010-00001, Revision 38, Savannah River Remediation, April 18, 2012.

2 Starks, J. B., “The PUREX Process,” DPSPU 77-11-1, E. I. du Pont de Nemours \& Co., January 1977.

3 Gray, L. W. and K. W. MacMurdo, "Trace Elements Retained in Washed Nuclear Fuel Reprocessing Solvents,” DP-1527, E. I. du Pont de Nemours \& Co., September 1979.

4 Gray, L. W., E. L. Christensen, J. D. Navratil, and W. W. Schulz, "Present Status and Future Direction of Plutonium Process Chemistry," DP-MS-82-63, E. I. du Pont de Nemours \& Co., September 1982.

5 Cavin, W. S., "Separations Waste Sludge Spreadsheet," WSRC-TR-93-135, Revision 1, Westinghouse Savannah River Company, July 29, 1993.

6 Eibling, R. E., "SRS High Level Sludge Chemistry," WSRC-MS-2007-00073, Westinghouse Savannah River Company, March 28, 2007. 
$7 \quad$ Cavin, W. S., "Fresh Waste Receipts," WSRC-TR-93-426, Revision 1, Westinghouse Savannah River Co., January 24, 1994.

$8 \quad$ Cavin, W. S., "Waste Transfers and Miscellaneous Additions," WSRC-TR-93425, Revision 1, Westinghouse Savannah River Co., January 24, 1994.

9 Chandler, M. C., "Estimated Sludge Inventory for Individual Tanks," WSRC-TR94-0191, Revision 1, Westinghouse Savannah River Company, August 1994.

10 Hester, J. R., "Sludge 1.5 Correction for Ironless Canyon Transfers," X-ESR-G00005, Westinghouse Savannah River Company, February 3, 2005.

11 Georgeton, G. K. and J. R. Hester, "Characterization of Radionuclides in HLW Sludge Based on Isotopic Distribution in Irradiated Assemblies," WSRC-TR-940562, Revision 1, Westinghouse Savannah River Co., January 27, 1995.

12 "Sludge 1.5 Spreadsheets," WG08 server, Savannah River Remediation, July 2012.

13 Monier, J. A. and T. C. Evans, "Thoria Powder Specifications, EM Specification 162,” E. I. du Pont de Nemours \& Company, Savannah River Site, Aiken, SC, May 10, 1968.

14 Walker, D. D. and M. A. Schmitz, "Technical Data Summary: In-Tank Precipitation Processing of Soluble High-Level Waste," DPSTD-84-103, E. I. du Pont de Nemours \& Co., May 1984.

15 Pike, J. A. and J. M. Gillam, "Flowsheet for Aluminum Removal from Sludge Batch 6," SRNL-STI-2008-00389, Savannah River National Laboratory, December 2008.

16 Pike, J. A., "Evaluation of Low Temperature Aluminum Dissolution in Tank 51," SRNS-STI-2008-00021, Savannah River Nuclear Solutions, September 2008.

17 Keefer, M. T., S. G. Campbell, D. J. Clark, and M. D. Buxton, "Sludge Heel Removal by Aluminum Dissolution at Savannah River Site," WM2012 Conference, Phoenix, AZ, February 26 - March 1, 2012.

18 "Industrial Wastewater Closure Module for the Liquid Waste Tanks 18 and 19, FArea Tank Farm SRS," SRR-CWDA-2010-0003, Revision 2, Savannah River Remediation, January 2012.

19 Poirier, M. R., D. T. Herman, F. F. Fondeur, J. M. Pareizs, M. S. Hay, B. J. Wiersma, K. P. Crapse, T. B. Peters, S. D. Fink, and G. D. Thaxton, "Removing Sludge Heels from Savannah River Site Waste Tanks by Oxalic Acid Dissolution," WM2009 Conference, Pheonix, AZ, March 1-5, 2009. 
20 King, W. D. and M. S. Hay, "Alternative Enhanced Chemical Cleaning: Basic Studies Results FY09," SRNL-STI-2009-00791, Savannah River National Laboratory, February 2010.

21 Lambert, D. P., “Analysis of June 2009 Tank 7 3-L Sample,” SRNL-L3100-200900232, Revision 1, Savannah River National Laboratory, September 2009.

22 Reboul, S. H., M. S. Hay, K. E. Zeigler, and M. E. Stone, “Tank 12 Sludge Characterization and Aluminum Dissolution Demonstration," SRNL-STI-200900180, Savannah River National Laboratory, March 2009.

23 Hay, M. S., P. E. O'Rourke, and H. M. Ajo, "Scanning Electron Microscopy and $X$-Ray Diffraction Analysis of Tank 18 Samples,” SRNL-STI-2012-00123, Savannah River National Laboratory, March 2012.

24 Hay, M. S., “Analysis of Tank 19F by X-Ray Diffraction,” SRT-LWP-97-111, Savannah River National Laboratory, September 1997.

25 Hay, M. S. and N. E. Bibler, "Characterization and Sludge Washing Studies of Tank 42H Sludge,” WSRC-RP-94-730, Westinghouse Savannah River Co., July 1994.

26 Hay, M. S., J. M. Pareizs, C. J. Bannochie, M. E. Stone, D. R. Click, and D. J. McCabe, "Characterization and Aluminum Dissolution Demonstration with a 3 Liter Tank 51H Sample," WSRC-STI-2007-00697, Westinghouse Savannah River Co., February 2008.

27 Anders, E., The Radiochemistry of Technetium, NAS-NS 3021, National Academy of Sciences, November 1960.

28 Krupka, K. M. and R. J. Serne, "Geochemical Factors Affecting the Behavior of Antimony, Cobalt, Europium, Technetium, and Uranium in Vadose Sediments," PNNL-14126, Pacific Northwest National Laboratory, December 2002.

29 Fowler, J. R., B. A. Hamm, and J. R. Cook, "Technetium-99 in SRP High-level Waste and Saltstone," DPST-84-334, E. I. du Pont de Nemours \& Co., February 24, 1984.

30 Peretyazhko, T., J. M. Zachara, S. M. Heald, B. H. Jeon, R. K. Kukkadapu, C. Liu, D. Moore, and C. T. Resch, "Heterogeneous Reduction of Tc(VII) by Fe(II) at the Solid-Water Interface," Geochim. Cosmochim. Acta, 72, 1521-1539, 2009. 
31 Li, D. and D. I. Kaplan, "Literature Review on the Sorption of Plutonium, Uranium, Neptunium, Americium, and Technetium to Corrosion Products on Waste Tank Liners," SRNL-STI-2012-00040, Savannah River National Laboratory, February 2012.

32 Shuh, D. K., W. W. Lukens, and C. J. Burns, "Final Report: Research Program to Investigate the Fundamental Chemistry of Technetium," Project EMSP-73778, Lawrence Berkeley National Laboratory, 2003.

33 Norato, M. A., S. D. Fink, F. F. Fondeur, G. F. Kessinger, R. A. Pierce, and D. D. Walker, "Demonstration of Caustic-Side Solvent Extraction with Optimized Solvent in the 2-cm Centrifugal Contactor Apparatus Using Dissolved Salt Cake from Tank 37H," WSRC-TR-2002-00307, Revision 1, Westinghouse Savannah River Co., September 2002.

34 Bibler, N. E., T. L. Fellinger, and D. T. Hobbs, "Technetium-99 Behavior in Savannah River Site High Level Waste Sludges during Waste Processing," WSRC-TR-2004-00196, Westinghouse Savannah River Co., April 2004.

35 Cantrell, K. J., K. M. Krupka, W. J. Deutsch, and M. J. Lindberg, "Residual Waste from Hanford Tanks 241-C-203 and 241-C-204 2. Contaminant Release Model," Environ. Sci. Technol., 40, 3755-3761, 2006.

36 Poirier, M. R., F. F. Fondeur, D. M. Missimer, M. E. Summer, and S. D. Fink, "Analysis of Solids Remaining Following Chemical Cleaning of Tank 5F," SRNLSTI-2010-00199, Savannah River National Laboratory, March 2010.

37 Poirier, M. R., F. F. Fondeur, D. M. Missimer, M. E. Summer, and S. D. Fink, "Analysis of Solids Remaining Following Chemical Cleaning of Tank 6F," SRNLSTI-2009-00816, Savannah River National Laboratory, February 2010.

38 Oji, L. N., D. D. DiPrete, and C. J. Coleman, "Characterization of Additional Tank 19F Floor Samples,” SRNL-STI-2010-00439, Savannah River National Laboratory, August 2010. 


\section{Distribution:}

H. H. Burns, 773-41A

D. A. Crowley, 773-43A

W. B. Dean, 705-1C

M. E. Denham, 773-42A

S. D. Fink, 773-A

G. P. Flach, 773-42A

K. M. Fox, 999-W

B. J. Giddings, 786-5A

C. C. Herman, 999-W

D. T. Hobbs, 773-A

S. Hommel, 705-1C
M. H. Layton, 705-1C

M. J. Mahoney, 705-1C

S. L. Marra, 773-A

A. M. Murray, 773-A

F. M. Pennebaker, 773-42A

S. H. Reboul, 773-A

K. H. Rosenberger, 705-1C

J. H. Scogin, 773-A

S. A. Thomas, 705-1C

A. W. Wiggins, 705-1C

W. R. Wilmarth, 773-A 